Pacific Journal of Mathematics

THE ADAMS SPECTRAL SEQUENCE OF THE REAL
PROJECTIE SPACES 


\section{THE ADAMS SPECTRAL SEQUENCE OF THE REAL PROJECTIVE SPACES}

\section{Ralph L. Cohen, Wen-Hsiung Lin and Mark E. Mahowald}

In this paper we study the mod 2 Adams spectral sequence for the infinite real projective space $P=\mathbf{R} P^{\infty}$.

We recall ([1]) that the spectral sequence starts with

$$
E_{2}^{s, t}=\operatorname{Ext}_{A}^{s, t}\left(\widetilde{H}^{*}(P), \mathrm{Z} / 2\right)
$$

and converges to the stable homotopy ${ }_{2} \pi_{*}^{s}(P)=\pi_{*}^{s}(P)$ where $A$ denotes the mod 2 Steenrod algebra and $\tilde{H}^{*}(P)$ is the reduced mod 2 cohomology of $P$. We simply write $\operatorname{Ext}_{A}^{s, t}(P)$ for $\operatorname{Ext}_{A}^{s, t}\left(\tilde{H}^{*}(P), \mathrm{Z} / 2\right)$ and occasionally we abbreviate by ASS for "Adams spectral sequence".

Roughly, our main results consist of (1) a complete description of $\operatorname{Ext}_{A}^{s, *}(P)$ for $0 \leq s \leq 2$ and also for $s=3$ modulo indecomposable elements, (2) the determination of which classes in a substantial portion of $\operatorname{Ext}_{A}^{1, *}(P)$ can detect homotopy elements in $\pi_{*}^{s}(P)$ (Adams's Hopf invariant Theorem solves the problem for $\left.\operatorname{Ext}_{A}^{0, *}(P)\right)$ and (3) the construction of some infinite families in $\pi_{*}^{s}(P)$ at low Adams filtrations analogous to the ones in the 2-adic stable homotopy of spheres ${ }_{2} \pi_{*}^{s}$ constructed in [9], [12] and [18], [22].

These Ext calculations were necessary in the work on the Kervaire invariant in [12]. The results are not surprising, but proving them is surprisingly difficult. In particular we make use of a calculational method that may be of independent interest.

To precisely state the results we first recall that the cohomology $\operatorname{Ext}_{A}^{*, *}=\operatorname{Ext}_{A}^{*, *}(\mathbf{Z} / 2, \mathbf{Z} / 2)$ of the Steenrod algebra $A$ is a commutative bigraded algebra over $\mathbf{Z} / 2$ and that $\operatorname{Ext}_{A}^{*, *}$ for $0 \leq s \leq 3$ is generated by $h_{i} \in \operatorname{Ext}_{A}^{1,2^{i}}(i \geq 0)$ and $c_{i} \in \operatorname{Ext}_{A}^{3,2^{i+3}+2^{i+1}+2^{i}}$ with relations $h_{i} h_{i+1}=$ $0, h_{i+1}^{3}=h_{i}^{2} h_{i+2}$ and $h_{i} h_{i+2}^{2}=0$ where $h_{i}$ corresponds to the Steenrod square $\mathrm{Sq}^{2^{i}} \in A$. The $\bmod 2$ cohomology $H^{*}(P)$ is a polynomial algebra $Z / 2[x]$ in one variable $x$ with $\operatorname{deg} x=1$ on which $A$ acts by $\mathrm{Sq}^{k} x^{i}=\left(\begin{array}{c}i \\ k\end{array}\right) x^{i+k}$. One easily proves that $\left\{x^{2^{i}-1} \mid i \geq 1\right\}$ is a minimal set of generators of $\tilde{H}^{*}(P)$ over $A$. The non-zero class in $\operatorname{Ext}_{A}^{0,2^{i}-1}(P)=$ $\mathrm{Z} / 2$ corresponding to $x^{2^{i}-1}$ is denoted by $\hat{h}_{i}$. The first part of the 
following theorem is therefore clear but for completeness and for our computational purpose later we include it in the statement.

THEOREM 1.1. (1) $\left\{\hat{h}_{i} \mid i \geq 1\right\}$ is a $\mathbf{Z} / 2$-base for $\operatorname{Ext}_{A}^{0, *}(P)$.

(2) $\operatorname{Ext}_{A}^{1, *}(P)$ is generated by $\hat{h}_{i} h_{j}$ with relations $\hat{h}_{i} h_{i-1}=0(i \geq 1)$.

(3) $\operatorname{Ext}_{A}^{2, *}(P)$ is generated by $\hat{h}_{i} h_{j} h_{k}$ and $\hat{c}_{i} \in \operatorname{Ext}_{A}^{2,2^{i+3}+2^{i+1}+2^{i}-1}(P)$ $(i \geq 0)$ with the relations coming from $\mathrm{Ext}_{A}^{* * *}$, the relations implied by (2) and the relations

$$
\begin{array}{ll}
\hat{h}_{i+2} h_{i}^{2}=\hat{h}_{i+1} h_{i+1}^{2} & (i \geq 0), \\
\hat{h}_{i+2} h_{i+2} h_{i}=0 & (i \geq 0) .
\end{array}
$$

(4) The products $\hat{h}_{i} h_{j} h_{k} h_{l}, \hat{h}_{i} c_{j}, \hat{c}_{i} h_{j}$ in $\operatorname{Ext}_{A}^{3, *}(P)$ are subject only to the relations from $\mathrm{Ext}_{A}^{*, *}$, the relations implied by (2) and (3) together with the following relations:

$$
\begin{gathered}
\hat{h}_{i+3} h_{i}^{2} h_{i+3}=0, \quad \hat{h}_{i+2} h_{i} h_{i+3}^{2}=0, \\
\hat{h}_{i} c_{j}=0 \text { for } i=j+2 \text { and } i=j+3, \\
\hat{c}_{i} h_{j}=0 \text { for } i=j-3, j-2, j \text { and } j+1, \\
\hat{c}_{i} h_{i+1}=\hat{h}_{i+1} c_{i} \neq 0 .
\end{gathered}
$$

THEOREM 1.2. In the Adams spectral sequence for $P$ there are the following differentials.

(1) $d_{2}\left(\hat{h}_{i} h_{j}\right)=\hat{h}_{i} h_{0} h_{j-1}^{2} \neq 0$ for $i=2,3$ and $j \geq 5$.

(2) $d_{2}\left(\hat{h}_{i} h_{j}\right)=\hat{h}_{i-1} h_{0} h_{j} h_{i-1} \neq 0$ for $i \geq 5, j=0 ; i \geq 6, j=2$ and $i \geq 7, j=3$.

(3) $d_{2}\left(\hat{h}_{i} h_{j}\right)=\hat{h}_{i-1} h_{0} h_{i-1} h_{j}+\hat{h}_{i} h_{0} h_{j-1}^{2} \neq 0$ for $i, j \geq 4$ with $i \neq$ $j, j+1$.

THEOREM 1.3. The following families in $\operatorname{Ext}_{A}^{*, *}(P)$ survive the Adams spectral sequence and so detect homotopy elements in $\pi_{*}^{s}(P)$.

(1) $\hat{h}_{1} h_{i}(i \geq 1)(2) \hat{h}_{i} h_{1}(i \geq 3)(3) \hat{h}_{2} h_{i}^{2}(i \neq 1)(4) \hat{h}_{i} h_{i} h_{2}(i \geq 5)$ (5) $\hat{h}_{i} c_{1}(i \neq 3,4)$.

We make some remarks on these results.

Theorem 1.2 is a consequence of Theorem 1.1 and Adams's Hopf invariant Theorem. Indeed, in the $\bmod 2$ ASS for ${ }_{2} \pi_{*}^{S}$ in which $\operatorname{Ext}_{A}^{* * *}$ is the $E_{2}$ term there are differentials $d_{2}\left(h_{i}\right)=h_{0} h_{i-1}^{2} \neq 0$ for $i \geq 4$ ([2]) while $h_{i}$, for $0 \leq i \leq 3$, are well known to detect homotopy elements. Let $\varphi: P \rightarrow S^{0}$ be the standard transfer map from the 
suspension spectrum of $P$ to the sphere spectrum $S^{0}$ ([15], [19]). The Kahn-Priddy Theorem ([15]) says that the induced map $\varphi_{\sharp}: \pi_{k}^{s}(P) \rightarrow$ ${ }_{2} \pi_{k}^{s}\left(S^{0}\right)={ }_{2} \pi_{k}^{s}$ is epimorphism for $k>0$. $\varphi$ also induces a map

$$
\varphi_{*}: \operatorname{Ext}_{A}^{s, t}(P) \rightarrow \operatorname{Ext}_{A}^{s+1, t+1}=\operatorname{Ext}_{A}^{s+1, t+1}\left(\tilde{H}^{*}\left(S^{0}\right)=\mathbf{Z} / 2, \mathbf{Z} / 2\right)
$$

in $E_{2}$ terms of the ASS's such that $\varphi_{*}\left(\hat{h}_{i}\right)=h_{i}$ and $\varphi_{*}\left(\hat{c}_{i}\right)=c_{i}([17]$, [19]). Since $\varphi_{*}$ commutes with $d_{2}$ and $\operatorname{Ext}_{A}^{2,2^{2}}(P)=\mathrm{Z} / 2$ is generated by $\hat{h}_{i-1} h_{0} h_{i-1}$ for $i \geq 4$ it follows that $d_{2}\left(\hat{h}_{i}\right)=\hat{h}_{i-1} h_{0} h_{i-1} \neq 0$ for $i \geq 4$ from which Theorem 1.2 follows.

The non-zero classes $\hat{h}_{i} h_{j}$ in $\operatorname{Ext}_{A}^{1, *}(P)$ not listed in (1.2) or (1.3) are classified in the following families:
(i) $\hat{h}_{i} h_{j}, i, j \leq 3$.
(ii) $\hat{h}_{2} h_{4}, \hat{h}_{3} h_{4}, \hat{h}_{4} h_{2}$.
(iii) $\hat{h}_{4} h_{4}, \hat{h}_{5} h_{5}$.
(iv) $\hat{h}_{4} h_{0}, \hat{h}_{5} h_{2}, \hat{h}_{5} h_{3}$.
(v) $\hat{h}_{6} h_{3}$.
(vi) $\hat{h}_{i} h_{i}, i \geq 6$.

The first five of these are known to detect or not to detect homotopy elements in $\pi_{*}^{s}(P):(\mathrm{i})$, (ii) and (iii) do while (iv) and (v) do not. Indeed, (i) is trivial and is well known, (ii) is proved in [19] and (iii) is a consequence of Kahn-Priddy Theorem and the results in [20], [4] where $h_{4}^{2}, h_{5}^{2}$ are proved to survive the ASS for $S^{0}$. For (iv), it is proved in [19] that, in the ASS for $P, d_{3}\left(\hat{h}_{4} h_{0}\right) \neq 0$ and $d_{4}\left(\hat{h}_{5} h_{2}\right) \neq 0$ and $d_{4}\left(\hat{h}_{5} h_{3}\right) \neq 0$. The fact that $(v)$ does not detect a homotopy class was proved by Mitchell (see [28]). For (vi) we refer to [3] for a discussion on this particular family.

One should compare Theorem 1.2 with the result in [21] where a similar situation in the spheres was analyzed.

Finally we remark on Theorem 1.3. In [9], [12], [18] and [22] the families $\left\{h_{i} h_{1} \mid i \geq 3\right\},\left\{h_{i}^{2} h_{2} \mid i \geq 5\right\},\left\{h_{i} c_{1} \mid i \geq 5\right\}$ in $\operatorname{Ext}_{A}^{*, *}$ are shown to detect homotopy elements in ${ }_{2} \pi_{*}^{s}$. From Theorem 1.1 we see, under the map $\varphi_{*}: \operatorname{Ext}_{A}^{* * *}(P) \rightarrow \operatorname{Ext}_{A}^{*+1, *+1}$, there correspond two families in $\operatorname{Ext}_{A}^{* * *}(P)$ to each of the families $\left\{h_{i} h_{1}\right\},\left\{h_{i}^{2} h_{2}\right\}$ and $\left\{h_{i} c_{1}\right\}$. Indeed, $\varphi_{*}\left(\hat{h}_{i} h_{1}\right)=\varphi_{*}\left(\hat{h}_{1} h_{i}\right)=h_{i} h_{1}, \varphi_{*}\left(\hat{h}_{2} h_{i}^{2}\right)=\varphi_{*}\left(\hat{h}_{i} h_{2} h_{i}\right)=h_{i}^{2} h_{2}, \varphi_{*}\left(\hat{c}_{1} h_{i}\right)=$ $\varphi_{*}\left(\hat{h}_{i} c_{1}\right)=h_{i} c_{1}$. By Kahn-Priddy Theorem, homotopy elements in ${ }_{2} \pi_{*}^{s}$ detected by $h_{i} h_{1}$ (resp. $h_{i}^{2} h_{2}, h_{i} c_{1}$ ) lift to elements in $\pi_{*}^{s}(P)$ which must be detected by $\hat{h}_{i} h_{1}$ or $\hat{h}_{1} h_{i}$ (resp. $\hat{h}_{2} h_{i}^{2}$ or $\hat{h}_{i} h_{i} h_{2}, \hat{h}_{i} c_{1}$ or $\hat{c}_{1} h_{i}$ ), and in general one does not know which of these two possibilities is the correct one. The result (1.3) shows that for the families $\left\{h_{i} h_{1}\right\}$ and $\left\{h_{i}^{2} h_{2}\right\}$ both choices are possible and for the family $\left\{h_{i} c_{1}\right\}$ one has a specific choice. If the strong Kervaire invariant conjecture (see 
[3]) can be shown true then the family $\left\{\hat{c}_{1} h_{i}\right\}$ can be easily shown to detect homotopy elements also. Otherwise we have no answer thus far about this family.

The family $\left\{\hat{h}_{1} h_{i}\right\}$ in Theorem 1.3 was already proved in [12] to detect homotopy elements. It is included here for comparison with $\left\{\hat{h}_{i} h_{1}\right\}$ and also serves as a preparatory statement for going on proving $(1.3)(3)$ as will be seen in $\S 3$.

This concludes our remarks.

Our work is therefore to prove Theorem 1.1 and Theorem 1.3. Theorem 1.1 is proved in $\S 2$ and Theorem 1.3 is proved in $\S 3$. The proof of Theorem 1.3 is shorter and one may proceed directly to $\S 3$ for the proof assuming the results in (1.1).

The proof of Theorem 1.1 is rather long. It is purely algebraic of course and is based on a lot of calculations. As mentioned earlier, a technical method, which later on is referred to as a "trick", is introduced to make these calculations tractable. In the beginning of the proof we give full details of our calculations. As the calculations progress we will begin omitting many of the details, as by then the reader should have no difficulty in using the "trick" to fill them in.

2. Proof of Theorem 1.1. Theorem 1.1 is proved by calculating with the lambda algebra $\Lambda$ of Kan et al. ([6]).

We begin by recalling that $\Lambda$ is a bigraded differential algebra over $\mathrm{Z} / 2$ generated by $\lambda_{i} \in \Lambda^{1, i}(i \geq 0)$ with relations

$$
\text { (a) } \lambda_{i} \lambda_{2 i+1+n}=\sum_{j \geq 0}\left(\begin{array}{c}
n-j-1 \\
j
\end{array}\right) \lambda_{i+n-j} \lambda_{2 i+1+j}
$$

The differential $\delta$ is given by

$$
\text { (b) } \delta\left(\lambda_{k}\right)=\sum_{j \geq 0}\left(\begin{array}{c}
k-j-1 \\
j+1
\end{array}\right) \lambda_{k-j-1} \lambda_{j}
$$

and $H^{s, t}(\Lambda, \delta) \simeq \operatorname{Ext}_{A}^{s, t+s}$. From (a) we see $\left\{\lambda_{i_{1}} \lambda_{i_{2}} \cdots \lambda_{i_{s}} \mid 2 i_{j} \geq i_{j+1}\right\}$ is a $Z / 2$-base for $\Lambda$. Such monomials are said to be admissible.

The class $h_{i} \in \operatorname{Ext}_{A}^{1,2^{i}}$ is represented by $\lambda_{2^{i}-1}$ and $c_{i} \in \operatorname{Ext}_{A}^{3,2^{i+3}+2^{i+1}+2^{i}}$ is represented by $\lambda_{2^{i+1}+2^{i}-1}\left(\lambda_{2^{i+2}-1}\right)^{2}$. Thus $h_{i} h_{j}$ for $0 \leq i \leq j$ (resp. $h_{i} h_{j} h_{k}, 0 \leq i \leq j \leq k$ ) is represented by $\lambda_{2^{i}-1} \lambda_{2^{\prime}-1}$ (resp. $\lambda_{2^{i}-1} \lambda_{2^{j}-1}$. $\left.\lambda_{2^{k}-1}\right)$. Note that we have chosen these representations in the arithmetic linear ordering of the subscripts. Most of these representations are inadmissible. They are admissible only in the cases $i=j$ and $i=j=k$. One advantage of taking such representations is that 
one recognizes immediately the relations $h_{i} h_{i+1}=0, h_{i}^{3}=h_{i-1}^{2} h_{i+1}$ and $h_{i} h_{i+2}^{2}=0$ in the lambda algebra. Indeed, it is easy to see from (a) that the relations $\lambda_{2^{i}-1} \lambda_{2^{i+1}-1}=0, \lambda_{2^{i-1}-1}^{2} \lambda_{2^{i+1}-1}=\lambda_{2^{i}-1}^{3}$ and $\lambda_{2^{i}-1}\left(\lambda_{2^{i+2}-1}\right)^{2}=0$ hold in $\Lambda$. Finally note that the representations for $c_{i}$ are admissible.

To compute $\operatorname{Ext}_{A}^{* * *}(P)$ we take $\tilde{H}_{*}(P)$, the $\bmod 2$ homology of $P$, and consider $\tilde{H}_{*}(P) \otimes \Lambda$ which is bigraded by $\left(\tilde{H}_{*}(P) \otimes \Lambda\right)^{s, t}=\sum_{k \geq 1} \tilde{H}_{k}(P) \otimes$ $\Lambda^{s, t-k} . \tilde{H}_{*}(P) \otimes \Lambda$ is a differential right $\Lambda$-module with differential $\delta$ given by

$$
\text { (c) } \delta\left(e_{k}\right)=\sum_{j \geq 0}\left(\begin{array}{c}
k-j-1 \\
j+1
\end{array}\right) e_{k-j-1} \otimes \lambda_{j}
$$

where $e_{k}$ is the generator of $\tilde{H}_{k}(P)=\mathbf{Z} / 2(k \geq 1)$. Then

$$
H^{s, t}\left(\tilde{H}_{*}(P) \otimes \Lambda, \delta\right) \cong \mathrm{Ext}_{A}^{s, t}(P) \quad([13]) .
$$

Define a filtration $\{F(i)\}_{i \geq 1}$ of the differential module $\tilde{H}_{*}(P) \otimes \Lambda$ by $F(i)=\sum_{k \leq i} e_{k} \otimes \Lambda$. Clearly $F(i) / F(i-1) \cong \Sigma^{i} \Lambda$. This filtration gives rise to a spectral sequence $\left\{E_{r}^{i, s, t}\right\}_{r \geq 1}$ with $\sum_{i \geq 1} E_{\infty}^{i, s, t} \cong \operatorname{Ext}_{A}^{s, t}(P)$ as a vector space over $Z / 2$ and

$$
\text { (d) } \quad E_{1}^{i, s, t}=H^{s, t}(F(i) / F(i-1)) \cong \mathrm{Ext}_{A}^{s, t+s-i} \text {. }
$$

For each $r \geq 1$ the differential $d_{r}$ of the spectral sequence goes from $E_{r}^{i, s, t}$ to $E_{r}^{i-r, s+1, t-1}$.

Our task is to make calculations with this spectral sequence. We need only compute the differentials $d_{r}: E_{r}^{i, s, t} \rightarrow E_{r}^{i-r, s+1, t-1}$ for $0 \leq$ $s \leq 2$. By (d) we see

$$
\begin{aligned}
& \left\{e_{i}=e_{i} \otimes 1 \mid i \geq 1\right\} \text { is a } \mathbf{Z} / 2 \text {-base for } E_{1}^{*, 0, *}, \\
& \left\{e_{i} h_{j}=e_{i} \otimes h_{j} \mid i \geq 1, j \geq 0\right\} \text { is a } \mathbf{Z} / 2 \text {-base for } E_{1}^{* 1, *}, \\
& \left\{e_{i} h_{j} h_{k} \mid i \geq 1,0 \leq j \leq k, h_{j} h_{k} \neq 0\right\} \text { is a } \mathbf{Z} / 2 \text {-base for } E_{1}^{*, 2, *}
\end{aligned}
$$

and

$$
\left\{e_{i} h_{j} h_{k} h_{l} \mid i \geq 1,0 \leq j \leq k \leq l, h_{j} h_{k} h_{l} \neq 0\right\} \cup\left\{e_{i} c_{j} \mid i \geq 1, j \geq 0\right\}
$$

$$
\text { is a } Z / 2 \text {-base for } E_{1}^{*, 3, *} \text {. }
$$

We begin with $d_{r}: E_{r}^{*, 0, *} \rightarrow E_{r}^{*, 1, *}$, the simplest differentials.

Note that each integer $i \geq 1$ can be expressed in the form $i=$ $2^{l}(2 k+1)-1$ for uniquely determined integers $l \geq 0$ and $k \geq 0$. Then we have the following differentials from $E_{r}^{*, 0, *}$ to $E_{r}^{*, 1, *}$.

$$
\begin{gathered}
d_{r}\left(e_{2^{\prime}-1}\right)=0 \text { for } r \geq 1, l \geq 1 . \\
e_{2^{\prime}(2 k+1)-1} \rightarrow e_{2^{l+1} k-1} h_{l} \text { for } k \geq 1 \text { and } l \geq 0 .
\end{gathered}
$$


Here " $e_{i} x \rightarrow e_{i-r} y$ " means that $e_{i} x$ and $e_{i-r} y$ both survive to $E_{r}^{*, *, *}$ and $d_{r}\left(e_{i} x\right)=e_{i-r} y$; in the case of (2.1.2), $x=1$ and $y=h_{l}$.

(2.1.1) is clear since $e_{2^{\prime}-1}$ are cycles in $\tilde{H}_{*}(P) \otimes \Lambda$. It is easy to see, from (c), that in $\tilde{H}_{*}(P) \otimes \Lambda$

$$
\delta\left(e_{2^{l}(2 k+1)-1}\right) \equiv e_{2^{l+1} k-1} \lambda_{2^{l}-1} \quad \bmod F\left(2^{l+1} k-2\right) \quad \text { for } k \geq 1
$$

and this is (2.1.2).

Theorem 1.1 (1) follows from (2.1.1) and (2.1.2); the class $\hat{h}_{i}$ is represented by $e_{2^{i}-1}$.

Next we compute $d_{r}: E_{r}^{*, 1, *} \rightarrow E_{r}^{*, 2, *}$. By (2.1.2) we need only consider $d_{r}\left(e_{2^{\prime}(2 k+1)-1} h_{j}\right)$ for $l \leq j$.

$$
d_{r}\left(e_{2^{\prime}-1} h_{j}\right)=0 \text { for } r \geq 1,1 \leq l \leq j .
$$

This is clear since $e_{2^{l}-1} \lambda_{2^{\jmath}-1} \in F\left(2^{l}-1\right)$ projects to $e_{2^{l}-1} h_{j} \in$ $H^{*}\left(F\left(2^{l}-1\right) / F\left(2^{l}-2\right)\right)$ and is a cycle.

(2.2.2) $e_{2^{l}(2 k+1)-1} h_{j} \rightarrow e_{2^{l+1} k-1} h_{l} h_{j}$

$$
\text { for } l<j-1 \text { or } l=j \text { and } k \geq 1 \text {. }
$$

A direct calculation as that for (2.1.2) proves (2.2.2). Or, this follows directly from (2.1.2) by the fact that all our differentials are linear over $\mathrm{Ext}_{A}^{*, *}$.

It remains to compute $d_{r}$ on classes of the form $e_{2^{l}(2 k+1)-1} h_{l+1}, k \geq$ 1. Let $2 k=2^{p}\left(2 k_{1}+1\right)$; so $p \geq 1, k_{1} \geq 0$. Thus

$$
e_{2^{l}(2 k+1)-1} h_{l+1}=e_{2^{l+p+1} k_{1}+2^{l+p}+2^{l}-1} h_{l+1} \text {. }
$$

(2.2.3) If $p \geq 2$, then

$$
e_{2^{l+p+1} k_{1}+2^{l+p}+2^{l-1}} h_{l+1} \rightarrow e_{2^{l+p+1} k_{1}+2^{l+p}-2^{l}-1} h_{l+1}^{2} .
$$

(2.2.4) If $p=1, k_{1}=2^{m}-1 \geq 0$ then

$$
d_{r}\left(e_{2^{l+2} k_{1}+2^{l+1}+2^{\prime}-1} h_{l+1}=e_{2^{l+2+m}-2^{l}-1} h_{l+1}\right)=0 \text { for } r \geq 1 \text {. }
$$

(2.2.5) If $p=1, k_{1}=2^{a+b-2} \bar{k}_{1}+2^{a}-1$ with $a \geq 0, b \geq 3$ and $\bar{k}_{1} \geq 1$ then

$$
\begin{aligned}
& e_{2^{l+2} k_{1}+2^{l+1}+2^{l}-1} h_{l+1}=e_{2^{l+a+b} \bar{k}_{1}+2^{l+a+2}-2^{l}-1} h_{l+1} \\
& \rightarrow \begin{cases}e_{2^{l+a+b} \bar{k}_{1}-2^{l}-1} h_{l+1} h_{l+a+2} & \text { for } a \geq 1 \\
e_{2^{l+b} \bar{k}_{1}-2^{l+1}-2^{l}-1} h_{l+2}^{2} & \text { for } a=0 .\end{cases}
\end{aligned}
$$

(2.2.3) again follows by a direct calculation as that for (2.1.2). However, in order to see what is really happening, we illustrate the calculation as follows. The class $e_{i=\left(2^{l+p+1} k_{1}+2^{l+p}+2^{l}-1\right)} \lambda_{2^{l+1}-1}$ in $F(i)$ projects 
to $e_{i} h_{l+1} \in H^{*}(F(i) / F(i-1))$. We call the former a representation in $F(i)$ of the latter. From (c) we calculate

$$
\begin{array}{lc}
\delta\left(e_{2^{l+p+1} k_{1}+2^{l+p}+2^{l}-1} \lambda_{2^{l+1}-1}\right) & \\
\equiv e_{2^{l+p+1} k_{1}+2^{l+p}-1} \lambda_{2^{l}-1} \lambda_{2^{l+1}-1}+ & e_{2^{l+p+1} k_{1}+2^{l+p}-2^{l}-1} \lambda_{2^{l+1}-1}^{2} \\
& \bmod F\left(2^{l+p+1} k_{1}+2^{l+p}-2^{l}-2\right) \\
\equiv e_{2^{l+p+1} k_{1}+2^{l+p}-2^{l}-1} \lambda_{2^{l+1}-1}^{2} & \bmod F\left(2^{l+p+1} k_{1}+2^{l+p}-2^{l}-2\right)
\end{array}
$$

since $\lambda_{2^{l}-1} \lambda_{2^{l+1}-1}=0$ in $\Lambda$. This proves (2.2.3). Note that here the condition $p \geq 2$ is needed in order to have the term $e_{2^{l+p+1} k_{1}+2^{l+p}-2^{l}-1} \lambda_{2^{l+1}-1}$ present in the expansion of $\delta\left(e_{2^{p+l+1} k_{1}+2^{l+p}+2^{l}-1}\right)$.

To show (2.2.4) we note the equation

$$
\begin{aligned}
(2.2 .4)^{*} \delta\left(e_{2^{l+2+m}+2^{l}-1}\right) \equiv e_{2^{l+2+m}-1} \lambda_{2^{l}-1}+e_{2^{l+2+m}-2^{l}-1} \lambda_{2^{l+1}-1} & \bmod F\left(2^{l+2+m}-2^{l}-2\right) .
\end{aligned}
$$

Since the second term on the right side of the equation represents $e_{2^{1+2+m}-2^{\prime}-1} h_{l+1}$ and the first term is a cycle in $\tilde{H}_{*}(P) \otimes \Lambda$ it follows that $e_{2^{l+2+m}-2^{l}-1} h_{l+1}$ is an infinite cycle in the spectral sequence. This proves (2.2.4).

The proof of (2.2.5) is a little harder and is postponed.

Now we conclude Theorem 1.1 (2) from the above differentials. From (2.1.2) and (2.2.1) we see $\hat{h}_{l} h_{j} \neq 0$ in $\operatorname{Ext}_{A}^{1, *}(P)$ for $1 \leq l \leq$ $j$. (2.1.2) shows that, for $l \geq j+1, e_{2^{l}-1} h_{j}$ is a boundary in the spectral sequence, but it does not necessarily follow that $\hat{h}_{l} h_{j}$ is zero in $\operatorname{Ext}_{A}^{1, *}(P)$. It only implies that $\hat{h}_{l} h_{j}$ has filtration less than $2^{l}-1$. In fact, for $l>j+1$, the equation $(2.2 .4)^{*}$ shows that $\hat{h}_{l} h_{j}$ has filtration $2^{l}-2^{j}-1$ and there it projects to

$$
e_{2^{\prime}-2^{\jmath}-1} h_{j+1} \in H^{*}\left(F\left(2^{l}-2^{j}-1\right) / F\left(2^{P}-2^{j}-2\right)\right) ;
$$

in particular $\hat{h}_{l} h_{j}$ is non-zero in $\operatorname{Ext}_{A}^{1, *}(P)$. This fails for $l=j+1$. Indeed, in $\tilde{H}_{*}(P) \otimes \Lambda$

$$
\delta\left(e_{2^{j+1}+2^{j-1}}\right)=e_{2^{j+1}-1} \lambda_{2^{j-1}} .
$$

Thus $\hat{h}_{j+1} h_{j}=0$. By (2.2.2), (2.2.3) and (2.2.5) there are no other surviving elements in $E_{\infty}^{*, 1, *}$. This proves Theorem 1.1(2).

It is not easy to see the differentials $(2.2 .5)$ by direct calculations as that for (2.2.3). To tackle (2.2.5) we will introduce a method which not only gives a quick proof for these differentials but is also efficient in recognizing more complicated differentials later. 
Recall that the set of all the admissible monomials in the $\lambda_{i}$ 's is a $Z / 2$-base for $\Lambda$. Define an increasing filtration $\{\Lambda(i)\}_{i \geq 0}$ of the differential algebra $\Lambda$ by $\Lambda(i)=\left\{\lambda_{i_{1}} \cdots \lambda_{i_{s}} \mid\left(i_{1}, \ldots, i_{s}\right)\right.$ admissible and $\left.i_{1} \leq i\right\}$. Each $\Lambda(i)$ is indeed closed under $\delta([6])$. There is a short exact sequence

$$
0 \rightarrow \Lambda(i-1) \stackrel{e}{\rightarrow} \Lambda(i) \stackrel{h}{\rightarrow} \Sigma^{i} \Lambda(2 i) \rightarrow 0
$$

where $e$ is the inclusion map and $h$ is given by

$$
h\left(\lambda_{i_{1}} \lambda_{i_{2}} \cdots \lambda_{i_{s}}\right)= \begin{cases}\lambda_{i_{2}} \cdots \lambda_{i_{s}} & \text { if } i_{1}=i \\ 0 & \text { if } i_{1}<i .\end{cases}
$$

Thus $\Lambda(i) / \Lambda(i-1) \cong \Sigma^{i} \Lambda(2 i)$ as a chain complex. This filtration gives rise to a spectral sequence $\left\{\bar{E}_{r}^{i, s, t}\right\}_{r \geq 1}$ with $\sum_{i \geq 1} \bar{E}_{\infty}^{i, s, t} \cong \operatorname{Ext}_{A}^{s, t}$ as a vector space over $Z / 2$ and

$$
\bar{E}_{1}^{i, s, t}=H^{s, t}(\Lambda(i) / \Lambda(i-1)) \cong H^{s, t-i}(\Lambda(2 i)) .
$$

For each $r \geq 1$ the differential $\bar{d}_{r}$ of the spectral sequence goes from $\bar{E}_{r}^{i, s, t}$ to $\bar{E}_{r}^{i-r, s+1, t-1}$.

The following is the basis of the to-be-introduced method.

THEOREM 2.3. There is a chain map $\phi: \Lambda \rightarrow \tilde{H}_{*}(P) \otimes \Lambda$ (but not a differential $\Lambda$-map) such that

(1) $\phi(\Lambda(i)) \subseteq F(i)$ for all $i \geq 0$.

(2) $\phi\left(\lambda_{i_{1}} \lambda_{i_{2}} \cdots \lambda_{i_{s}}\right)=e_{i_{1}} \otimes \lambda_{i_{2}} \cdots \lambda_{i_{s}} \bmod F\left(i_{1}-1\right)$ for $\left(i_{1}, i_{2}, \ldots, i_{s}\right)$ admissible and $i_{1} \geq 1$.

This result is actually proved in [17] except that it is not stated in this context there. We will recall the proof at the end of this section.

It follows from (2.3) that the chain map $\phi$ induces a map of the spectral sequences $\left\{\phi_{r}: \bar{E}_{r}^{i, s, t} \rightarrow E_{r}^{i, s, t}\right\}$ where

$$
\phi_{1}: \bar{E}_{1}^{i, s, t} \cong H^{s, t-i}(\Lambda(2 i)) \rightarrow E_{1}^{i, s, t} \cong H^{s, t-i}(\Lambda) \cong \mathrm{Ext}_{A}^{s, t+s-i}
$$

is just the induced map of the inclusion $\Lambda(2 i) \rightarrow \Lambda$. It is easy to see that $H^{s, t-i}(\Lambda(2 i)) \cong H^{s, t-i}(\Lambda)$ if $t-i<2 i-1$; so in this range $\phi_{1}$ is an isomorphism. We call this range the "stable range".

Now we prove (2.2.5). Recall that we want to compute

$$
d_{r}\left(e_{2^{l+a+b} \bar{k}_{1}+2^{l+a+2}-2^{l}-1} h_{l+1}\right)
$$

where $a \geq 0, b \geq 3$ and $\bar{k}_{1} \geq 1$. Let $i=2^{l+a+b} \bar{k}_{1}+2^{l+a+2}-2^{l}-1$. Then $2^{l+1}-1<2 i-1$, and so we may consider

$$
\lambda_{i} h_{l+1} \in H^{*}(\Lambda(i) / \Lambda(i-1)) \text {. }
$$


Our method of computing $d_{r}\left(e_{i} h_{l+1}\right)$ is to compute $\bar{d}_{r}\left(\lambda_{i} h_{l+1}\right)$ and then apply the equation

$$
d_{r}\left(e_{i} h_{l+1}\right)=\phi_{r} \bar{d}_{r}\left(\lambda_{i} h_{l+1}\right)
$$

Clearly $\lambda_{i} \lambda_{2^{l+1-1}} \in \Lambda(i)$ is a representation of $\lambda_{i} h_{l+1}$. The key point of the method is that the inadmissible monomial

$$
\lambda_{2^{l}-1} \lambda_{2^{l+a+b}} \bar{k}_{1}+2^{l+a+2}-1
$$

is also a representation in $\Lambda(i)$ of $\lambda_{i} h_{l+1}$ because

$$
\lambda_{2^{l}-1} \lambda_{2^{l+a+b}} \bar{k}_{1}+2^{l+a+2-1}=\lambda_{i} \lambda_{2^{l+1}-1} \quad \bmod \Lambda(i-1)
$$

(recall $i=2^{l+a+b} \bar{k}_{1}+2^{l+a+2}-2^{l}-1$ ), and it is easier to see the differential $\bar{d}_{r}\left(\lambda_{i} h_{l+1}\right)$ from this representation. In fact, we have

$$
\begin{aligned}
\delta & \left(\lambda_{2^{l}-1} \lambda_{2^{l+a+b} \bar{k}_{1}+2^{l+a+2}-1}\right) \\
& \equiv \lambda_{2^{l}-1} \lambda_{2^{l+a+b} \bar{k}_{1}-1^{2}} \lambda_{2^{l+a+2}-1} \\
& \equiv\left\{\begin{array}{lr}
\lambda_{2^{l+a+b} \bar{k}_{1}-2^{l}-1} \lambda_{2^{l+1}-1} \lambda_{2^{l+a+2}-1} \bmod \Lambda\left(2^{l+a+b} \bar{k}_{1}-2^{l}-2\right), \quad a \geq 1 \\
\lambda_{2^{l+b} \bar{k}_{1}-2^{l+1}-2^{l}-1}\left(\lambda_{2^{l+2}-1}\right)^{2} & \bmod \Lambda\left(2^{l+b} \bar{k}_{1}-2^{l+1}-2^{l}-2\right), \\
a & =0
\end{array}\right.
\end{aligned}
$$

Thus

$$
\lambda_{i} h_{l+1} \rightarrow \begin{cases}\lambda_{i-2^{l+a+2}} h_{l+1} h_{l+a+2}, & a \geq 1 \\ \lambda_{i-2^{l+2}-2^{l+1}} h_{l+2}^{2}, & a=0\end{cases}
$$

So

$$
e_{i} h_{l+1} \rightarrow \begin{cases}e_{i-2^{l+a+2}} h_{l+1} h_{l+a+2}, & a \geq 1, \\ e_{i-2^{l+2}-2^{l+1}} h_{l+2}^{2}, & a=0 .\end{cases}
$$

This proves (2.2.5).

We proceed to compute $d_{r}: E_{r}^{*, 2, *} \rightarrow E_{r}^{*, 3, *}$, the last set of differentials. By (2.2.2) we need only consider $d_{r}\left(e_{2^{\prime}(2 n+1)-1} h_{j} h_{k}\right)$ for $l \leq j$. By (2.2.3) and (2.2.5) we may impose some other restrictions on the subscripts of these elements when $l=j-1$ or $j-2$. These restrictions will be recalled at proper time.

To prove Theorem 1.1(3) it suffices to know which of these nonboundary elements are infinite cycles.

Proposition 2.4. The following elements in the first column are infinite cycles and form a $\mathrm{Z} / 2$-base for $E_{\infty}^{*, 2, *}$; they represent the elements 
in $\operatorname{Ext}_{A}^{2, *}(P)$ listed in the second column.

(1) $e_{2^{\prime}-1} h_{j} h_{k} \longleftrightarrow \hat{h}_{l} h_{j} h_{k}$,

$$
1 \leq l \leq j \text { and } j<k-1 \text { or } j=k \text {. }
$$

(2) $e_{2^{j+1}+2^{j}-1} h_{j+2}^{2} \longleftrightarrow \hat{c}_{j}, \quad j \geq 0$

(3) $e_{2^{m}-2^{j-1}} h_{j}^{2} \longleftrightarrow \hat{h}_{m} h_{j-1}^{2}, \quad j \geq 1$ and $j+2 \leq m$.

(4) $e_{2^{m}-2^{j-1}-1} h_{j} h_{k} \longleftrightarrow \hat{h}_{m} h_{j-1} h_{k}$, $j \geq 1$ and $j+1 \leq m \leq k, j<k-1$.

(5) $e_{2^{m+t}-2^{m}-2^{j-1}-1} h_{j} h_{m+1} \longleftrightarrow \hat{h}_{m+t} h_{j-1} h_{m}$,

$$
j \geq 1, t \geq 2 \text { and } j+1 \leq m \text {. }
$$

We prove (2.4) excluding "they form a Z/2-base for $E_{\infty}^{*, 2, *}$ " which will be clear after we compute non-trivial differentials on the remaining elements later.

First of all, a check against (2.2.2), (2.2.3) and (2.2.5) shows that the elements in the first column of (2.4) are non-boundary elements.

$(2.4)(1)$ is clear. By a direct calculation we see $\delta\left(e_{2^{j+1}+2 \jmath-1} \lambda_{2^{j+2}-1}^{2}\right)=$ 0 in $\tilde{H}_{*}(P) \otimes \Lambda$. Let $\hat{c}_{j}=\left\{e_{2^{\jmath+1}+2^{\jmath}-1} \lambda_{2^{\jmath+2}-1}^{2}\right\} \in \operatorname{Ext}_{A}^{2,2^{j+3}+2^{j+1}+2^{\jmath}-1}(P)$. Then $\hat{c}_{j}$ projects to $e_{2^{j+1}+2^{j}-1} h_{j+2}^{2}$ in the spectral sequence. This proves $(2.4)(2) .(2.4)(4)$ follows from the equation

$$
\begin{aligned}
& \delta\left(e_{2^{m}+2^{j-1}-1} \lambda_{2^{k}-1}\right) \\
& \quad \equiv e_{2^{m}-2^{j-1}-1} \lambda_{2^{j}-1} \lambda_{2^{k}-1}+e_{2^{m}-1} \lambda_{2^{j-1}-1} \lambda_{2^{k}-1} \bmod F\left(2^{m}-2^{j-1}-2\right) .
\end{aligned}
$$

To prove (2.4)(3) and (5) we use the "trick" that we tackled with (2.2.5). Note that in both cases we are in the stable range.

For (2.4)(3) consider the equation

$$
\lambda_{2^{m}-2^{j}-1} \lambda_{2^{j}-1}^{2} \equiv \lambda_{2^{j-1}-1}^{2} \lambda_{2^{m}-1} \quad \bmod \Lambda\left(2^{m}-2^{j}-2\right)
$$

in $\Lambda$. Since $\operatorname{Ext}_{A}^{*, *}$ is commutative there exists $K \in \Lambda$ such that

$$
\delta(K)=\lambda_{2^{m}-1} \lambda_{2^{j-1}-1}^{2}+\lambda_{2^{j-1}-1}^{2} \lambda_{2^{m}-1} .
$$

Thus $\lambda_{2^{m}-2^{j}-1} \lambda_{2^{j}-1}^{2}+\delta(K) \equiv \lambda_{2^{m}-1} \lambda_{2^{j-1}-1}^{2} \bmod \Lambda\left(2^{m}-2^{j}-2\right)$. Note that both $\left(2^{m}-2^{j}-1,2^{j}-1,2^{j}-1\right)$ and $\left(2^{m}-1,2^{j-1}-1,2^{j-1}-1\right)$ are admissible. Applying the chain map $\phi$ in Theorem 2.3 to this equation we get

$$
e_{2^{m}-2^{j}-1} \lambda_{2^{\prime}-1}^{2}+\delta(\phi(K)) \equiv e_{2^{m}-1} \lambda_{2^{j-1}}^{2} \quad \bmod F\left(2^{m}-2^{j}-2\right) .
$$

This proves $(2.4)(3)$. 
For $(2.4)(5)$ consider the equations

$$
\begin{aligned}
\lambda_{2^{m+1}-2^{m}-2^{j-1}-1} \lambda_{2^{j}-1} \lambda_{2^{m+1}-1} \equiv \lambda_{2^{j-1}-1} \lambda_{2^{m+t}-2^{m}-1} \lambda_{2^{m+1}-1} & \\
& \bmod \Lambda\left(2^{m+t}-2^{m}-2^{j-1}-2\right)
\end{aligned}
$$

and

$$
\begin{aligned}
& \delta\left(\lambda_{2^{j-1}-1} \lambda_{2^{m+t}+2^{m}-1}\right)+\delta\left(K^{\prime}\right) \\
& \equiv \lambda_{2^{j-1}-1^{2}} \lambda_{2^{m+l}-2^{m}-1} \lambda_{2^{m+1}-1}+\lambda_{2^{m+t}-1} \lambda_{2^{j-1}-1} \lambda_{2^{m}-1} \\
& \bmod \Lambda\left(2^{m+t}-2^{m}-2^{j-1}-2\right)
\end{aligned}
$$

where

$$
\delta\left(K^{\prime}\right)=\lambda_{2^{\prime-1}-1} \lambda_{2^{m+t}-1} \lambda_{2^{m}-1}+\lambda_{2^{m+l}-1} \lambda_{2^{j-1}-1} \lambda_{2^{m}-1}
$$

So

$$
\begin{aligned}
& \delta\left(\lambda_{2^{j-1}-1} \lambda_{2^{m+l}+2^{m}-1}\right)+\delta\left(K^{\prime}\right) \\
& \equiv \lambda_{2^{m+t}-2^{m}-2^{j-1}-1} \lambda_{2^{j-1}} \lambda_{2^{m+1}-1}+\lambda_{2^{m+t}-1} \lambda_{2^{j-1}-1} \lambda_{2^{m}-1} \\
& \bmod \Lambda\left(2^{m+t}-2^{m}-2^{j-1}-2\right) .
\end{aligned}
$$

Again this implies

$$
\begin{aligned}
& \delta\left(\phi\left(\lambda_{2^{j-1}-1} \lambda_{2^{m+l}+2^{m}-1}\right)\right)+\delta\left(\phi\left(K^{\prime}\right)\right) \\
& \equiv e_{2^{m+l}-2^{m}-2^{\jmath-1}-1} \lambda_{2^{\jmath}-1} \lambda_{2^{m+1}-1}+e_{2^{m+l}-1} \lambda_{2^{\jmath-1}-1} \lambda_{2^{m}-1} \\
& \bmod F\left(2^{m+t}-2^{m}-2^{j-1}-2\right) .
\end{aligned}
$$

This proves $(2.4)(5)$.

To complete the proof of Theorem 1.1(3) (assuming the whole (2.4)) it remains to show the relations

$$
\hat{h}_{i+2} h_{i}^{2}=\hat{h}_{i+1} h_{i+1}^{2} \quad \text { and } \quad \hat{h}_{i+2} h_{i+2} h_{i}=0
$$

in $\operatorname{Ext}_{A}^{2, *}(P)$. As suggested by (2.2.2) we get these relations by the equations

$\delta\left(e_{2^{i+2}+2^{i}-1} \lambda_{2^{i}-1}\right)+\delta\left(e_{2^{i+1}+2^{i}-1} \lambda_{2^{i+1}+2^{i}-1}\right)=e_{2^{i+2}-1} \lambda_{2^{i}-1}^{2}+e_{2^{i+1}-1^{1}} \lambda_{2^{i+1}-1}^{2}$

and

$$
\delta\left(e_{2^{i+2}+2^{i}-1} \lambda_{2^{i+2}-1}\right)=e_{2^{i+2}-1} \lambda_{2^{i}-1} \lambda_{2^{i+2}-1}
$$

in $\tilde{H}_{*}(P) \otimes \Lambda$. This proves Theorem $1.1(3)$.

The most difficult part of the work is to compute the differentials on the remaining non-boundary elements $e_{2^{l}(2 n+1)-1} h_{j} h_{k}$ where $l \leq j$. There are 16 sets of non-trivial differentials on these elements. We will first state them. Proofs will be given later. These differentials will be needed in the proof of Theorem 1.1(4). 
First assume $j=k$. In this case there are 8 sets of non-trivial differentials. We state these differentials in the cases $l=j, l=j-1$, $l=j-2$ and $l \leq j-3$. Some of these cases will be divided into subcases.

(2.5.1) If $l=j=0$ then $e_{2 n} h_{0}^{2} \rightarrow e_{2 n-1} h_{0}^{3}, n \geq 1$.

(2.5.2) If $l=j \geq 1$ and $n+1$ is odd, say $n=2 \bar{n}$ then

$e_{2^{j}(2 n+1)-1} h_{j}^{2}=e_{2^{j+2} \bar{n}+2^{j}-1} h_{j}^{2} \rightarrow e_{2^{j+2} \bar{n}-2^{j+1}-2^{j-1}-1} c_{j-1}, \quad \bar{n} \geq 1$.

(2.5.3) If $l=j \geq 1, n+1=4 \bar{n}+2$ then

$e_{2^{j}(2 n+1)-1} h_{j}^{2}=e_{2^{j+3} \bar{n}+2^{j+2}-2^{j}-1} h_{j}^{2} \rightarrow e_{2^{\jmath+3} \bar{n}-2^{j+1}-2^{\jmath}-2^{\jmath-1}-1} h_{j-1} h_{j+2}^{2}$, $\bar{n} \geq 1$.

(2.5.4) If $l=j \geq 1, n+1=2^{a+b} \bar{n}+2^{a}, a \geq 2$ and $b \geq 1$, then $e_{2^{j}(2 n+1)-1} h_{j}^{2}=e_{2^{j+a+b+1} \bar{n}+2^{j+a+1}-2^{j-1}} h_{j}^{2} \rightarrow e_{2^{j+a+b+1} \bar{n}-2^{j}-1} h_{j}^{2} h_{j+a+1}$, $\bar{n} \geq 1$.

Suppose $l=j-1 \geq 0$. Then by (2.2.3) we need only consider $n=2 \bar{n}$. In this case we have the following.

$$
e_{2^{j-1}(2 n+1)-1} h_{j}^{2}=e_{2^{j+1} \bar{n}+2^{j-1}-1} h_{j}^{2} \rightarrow e_{2^{j+1} \bar{n}-2^{j-1}-1} h_{j}^{3}, \quad \bar{n} \geq 1 .
$$

Suppose $l=j-2 \geq 0$. Then by (2.2.5) we need only consider $n=2 \bar{n}+1$ or $n=4 \bar{n}$.

(2.5.6) If $l=j-2, n=2 \bar{n}+1$ then

$$
e_{2^{j-2}(2 n+1)-1} h_{j}^{2}=e_{2^{j} \bar{n}+2^{j-1}+2^{j-2}-1} h_{j}^{2} \rightarrow e_{2^{j} \bar{n}-1} c_{j-2}, \quad \bar{n} \geq 1 .
$$

(2.5.7) If $l=j-2, n=4 \bar{n}$ then

$$
e_{2^{j-2}(2 n+1)-1} h_{j}^{2}=e_{2^{j+1} \bar{n}+2^{j-2}-1} h_{j}^{2} \rightarrow e_{2^{j+1} \bar{n}-2^{j}+2^{j-2}-1} h_{j}^{3}, \quad \bar{n} \geq 1 .
$$

(2.5.8) If $l \leq j-3$ then

$$
e_{2^{\prime}(2 n+1)-1} h_{j}^{2} \rightarrow e_{2^{l+1} n-1} h_{l} h_{j}^{2}, \quad n \geq 1
$$

Next we state the remaining 8 sets of differentials for the case $j<$ $k-1$.

(2.5.9) If $l=j$ then

$$
e_{2^{\prime}(2 n+1)-1} h_{j} h_{k} \rightarrow e_{2^{j+1} n-1} h_{j}^{2} h_{k}, \quad n \geq 1 .
$$

Suppose $l=j-1 \geq 0$. Then by (2.2.5) we need only consider $n$ such that

$2^{j-1}(2 n+1)=2^{m+1} \bar{n}+2^{m}-2^{j-1}$ where $j \leq m \leq k$ and $\bar{n} \geq 0$. 
The case $\bar{n}=0$ is already covered by (2.4); so can assume $\bar{n} \geq 1$. Let $\bar{n}=2^{p-1}(2 q+1), p \geq 1, q \geq 0$. Then

$$
2^{j-1}(2 n+1)-1=2^{p+m}(2 q+1)+2^{m}-2^{j-1}-1 .
$$

The next 6 differentials are stated for the case $l=j-1$ depending on various conditions on $j, m, k, p$ and $q$.

(2.5.10) If $m=j$ then

$$
\begin{aligned}
e_{2^{\jmath-1}(2 n+1)-1} h_{j} h_{k} & =e_{2^{p+\jmath}(2 q+1)+2^{\jmath-1}-1} h_{j} h_{k} \\
& \rightarrow e_{2^{p+\jmath}(2 q+1)-2^{\jmath-1}-1} h_{j}^{2} h_{k}, \quad p \geq 1, q \geq 0 .
\end{aligned}
$$

(2.5.11) If $j+1 \leq m<k-1$ or if $k=m \geq j+3$ then

$$
\begin{aligned}
& e_{2^{\jmath-1}(2 n+1)-1} h_{j} h_{k}=e_{2^{p+m}(2 q+1)+2^{m}-2^{j-1}-1} h_{j} h_{k} \\
& \quad \rightarrow\left\{\begin{array}{l}
e_{2^{p+m}(2 q+1)-2^{\jmath-1}-1} h_{j} h_{m} h_{k} \text { for } m \geq j+2, \quad p \geq 1 \\
e_{2^{p+m}(2 q+1)-2^{j+1}+2^{\jmath-1}-1} h_{j+1}^{2} h_{k} \text { for } m=j+1, \quad q \geq 0 .
\end{array}\right.
\end{aligned}
$$

(2.5.12) If $j+1 \leq m=k-1$ and $p \geq 2$ then

$$
\begin{aligned}
& e_{2^{j-1}(2 n+1)-1} h_{j} h_{k}=e_{2^{p+m}(2 q+1)+2^{m}-2^{j-1}-1} h_{j} h_{m+1} \\
& \quad \rightarrow\left\{\begin{array}{l}
e_{2^{p+m}(2 q+1)-2^{m}-2^{j-1}-1} h_{j} h_{m+1}^{2} \text { for } m \geq j+2, \\
e_{2^{p+j+1}(2 q+1)-2^{j+2}-2^{j-1}-1} c_{j} \text { for } m=j+1, \quad q \geq 0 .
\end{array}\right.
\end{aligned}
$$

(2.5.13) If $j+1 \leq m=k-1, p=1$ and $2 q+1=2^{t} \bar{q}+2^{t-1}-1$, $t \geq 3, \bar{q} \geq 1$ then

$$
\begin{aligned}
e_{2^{j-1}(2 n+1)-1} h_{j} h_{k} & =e_{2^{m+t+1} \bar{q}+2^{m+t}-2^{m}-2^{j-1}-1} h_{j} h_{m+1} \\
& \rightarrow e_{2^{m+l+1} \bar{q}-2^{m}-2^{j-1}-1} h_{j} h_{m+1} h_{m+t} .
\end{aligned}
$$

(2.5.14) If $j+1 \leq m=k-1, p=1,2 q+1=4 \bar{q}+1, \bar{q} \geq 1$ then

$$
\begin{aligned}
e_{2^{j-1}(2 n+1)-1} h_{j} h_{k} & =e_{2^{m+3} \bar{q}+2^{m+1}+2^{m}-2^{j-1}-1} h_{j} h_{m+1} \\
& \rightarrow e_{2^{m+3} \bar{q}-2^{m+1}-2^{m}-2^{j-1}-1} h_{j} h_{m+1}^{2} .
\end{aligned}
$$

(2.5.15) If $m=k=j+2$ then

$$
\begin{aligned}
e_{2^{\jmath-1}(2 n+1)-1} h_{j} h_{k} & =e_{2^{p+j+2}(2 q+1)+2^{\jmath+2}-2^{j-1}-1} h_{j} h_{j+2} \\
& \rightarrow e_{2^{p+j+2}(2 q+1)-2^{\jmath+1}-2^{\jmath}-2^{j-1}-1} h_{j+2}^{3}, \quad p \geq 1, q \geq 0 .
\end{aligned}
$$

Finally we have the following.

(2.5.16) If $l \leq j-2$ then

$$
e_{2^{l}(2 n+1)-1} h_{j} h_{k} \rightarrow e_{2^{l+1} n-1} h_{l} h_{j} h_{k}, \quad n \geq 1 \text {. }
$$


This completes the statements of the 16 sets of non-trivial differentials $d_{r}: E_{r}^{*, 2, *} \rightarrow E_{r}^{*, 3, *}$.

It is not difficult to check that the elements $e_{2^{\prime}(2 n+1)-1} h_{j} h_{k}$ considered in (2.5.1) through (2.5.16) together with those listed in Proposition 2.4 form a complete set of non-boundary elements. Also, distinct elements in the domain of (2.5.1) through (2.5.16) have distinct values. This proves the second conclusion of Proposition 2.4.

Some of these differentials are straightforward as (2.2.2) or (2.2.3). They are (2.5.1), (2.5.5), (2.5.6), (2.5.7), (2.5.8), (2.5.9) and (2.5.16).

For the rest, we need the "trick" in the proof of (2.2.5) to handle them. We are not going to do all the calculations. Rather, we just do for two of these. One of them is the hardest of all, and the calculation for the other is very typical for doing the remaining 7 differentials which we leave to the reader.

First, the easy one which we choose to be (2.5.2). Consider $e_{i} \lambda_{2^{\prime}-1}^{2}$, where $i=2^{j+2} \bar{n}+2^{j}-1$, a representation in $F(i)$ of $e_{i} h_{j}^{2}$. Since $\bar{n} \geq 1$, we are in the stable range. The "trick" is to replace $e$ by $\lambda$, that is, to consider $\lambda_{i} \lambda_{2^{\prime}-1}^{2}$, a representation in $\Lambda(i)$ of $\lambda_{i} h_{j}^{2} \in H^{*}(\Lambda(i) / \Lambda(i-1))$ and then consider the equation

$$
\lambda_{i} \lambda_{2^{j}-1}^{2} \equiv \lambda_{2^{j-1}-1}^{2} \lambda_{i+2^{\prime}} \quad \bmod \Lambda(i-1)
$$

which shows $\lambda_{2^{j-1}}^{2} \lambda_{i+2}$, is also a representation in $\Lambda(i)$ of $\lambda_{i} h_{j}^{2}$. We have

$$
\begin{aligned}
& \delta\left(\lambda_{2^{\jmath-1}-1}^{2} \lambda_{i+2^{\jmath}}\right)=\lambda_{2^{\jmath-1}-1}^{2} \lambda_{2^{\jmath+2} \bar{n}+2^{\jmath+1}-1} \\
& \equiv \lambda_{2^{\jmath-1}-1}^{2} \lambda_{2^{\jmath+2} \bar{n}-1} \lambda_{2^{\jmath+1}-1} \\
& \equiv \lambda_{2^{j-1}-1} \lambda_{2^{\jmath+2} \bar{n}-2^{\jmath+1}+2^{\jmath-1}-1} \lambda_{2^{j+1}-1}^{2} \quad\left(\text { since } \lambda_{2^{\jmath}-1} \lambda_{2^{\jmath+1}-1}=0\right) \\
& \equiv \lambda_{2^{\jmath+2} \bar{n}-2^{\jmath+1}-2^{j-1}-1} \lambda_{2^{\jmath}+2^{j-1}-1} \lambda_{2^{\jmath+1}-1}^{2} \quad\left(\text { since } \lambda_{2^{\jmath}-1} \lambda_{2^{\jmath+1}-1}^{2}=0\right) \\
& \bmod \Lambda\left(2^{j+2} \bar{n}-2^{j+1}-2^{j-1}-2\right) .
\end{aligned}
$$

This implies

$$
\left.\lambda_{i} h_{j}^{2} \rightarrow \lambda_{2^{\jmath+2} \bar{n}-2^{j+1}-2^{j-1}-1} c_{j-1} \quad \text { (in the spectral sequence }\left\{\bar{E}_{r}^{*, *, *}\right\}\right)
$$

and therefore

$$
e_{i} h_{j}^{2} \rightarrow e_{2^{\jmath+2} \bar{n}-2^{\jmath+1}-2^{\jmath-1}-1} c_{j-1} \quad \text { (by applying } \phi \text { in Theorem 2.3). }
$$

This proves (2.5.2). 
Next we do the calculation for the hard one which is (2.5.3). We directly begin with $\lambda_{i} \lambda_{2^{\prime}-1}^{2}$ and the equation

$$
\lambda_{i} \lambda_{2^{\jmath}-1}^{2} \equiv \lambda_{2^{\jmath-1}-1}^{2} \lambda_{2^{\jmath}+i} \quad \bmod \Lambda(i-1)
$$

where $i=2^{j+3} \bar{n}+2^{j+2}-2^{j}-1, \bar{n} \geq 1$. As above we compute

$$
\begin{aligned}
\delta & \left(\lambda_{2^{\jmath-1}-1}^{2} \lambda_{i+2^{\jmath}}=\lambda_{2^{\jmath-1}-1}^{2} \lambda_{2^{\jmath+3} \bar{n}+2^{\jmath+2}-1}\right) \\
& \equiv \lambda_{2^{\jmath-1}-1}^{2} \lambda_{2^{j+3} \bar{n}-1} \lambda_{2^{\jmath+2}-1} \\
& \equiv \lambda_{2^{j-1}-1} \lambda_{2^{\jmath+3} \bar{n}-2^{\jmath-1}-1} \lambda_{2^{\jmath}-1} \lambda_{2^{\jmath+2}-1} \\
& \equiv \lambda_{2^{j+3} \bar{n}-2^{\jmath-1}} \lambda_{2^{\jmath}-1}^{2} \lambda_{2^{i+2}-1}=\lambda_{2^{\jmath+3} \bar{n}-2{ }^{\jmath}-1} \lambda_{2^{\jmath+1}-1}^{3}
\end{aligned}
$$

where the equivalence " $\equiv$ " is yet to be determined. We pause at this point because the calculation seems to suggest

$$
e_{i} h_{j}^{2} \rightarrow e_{i-2^{j+2}} h_{j+1}^{3} \text {. }
$$

However, by (2.5.5), $e_{i-2^{\jmath+2}} h_{j+1}^{3}$ is already hit in the spectral sequence by an element of lower filtration, namely $e_{i-2^{j+1}} h_{j+1}^{2}$. So we have to take into account this differential also in order to get the correct answer. We need to pick up some other terms of lower filtration when we move $\lambda_{2^{j-1}-1}$ across $\lambda_{2^{j+3} \bar{n}-2^{j-1}-1}$ in the calculation above. Note that in $\Lambda, \lambda_{2^{\jmath}-1} \lambda_{2^{j+2}-1}=\lambda_{2^{\jmath+1}+2^{\jmath}-1} \lambda_{2^{\jmath+1}-1}$. The calculation now continues as follows.

$$
\begin{aligned}
& \delta\left(\lambda_{2^{\prime-1}-1}^{2} \lambda_{2^{\jmath+3} \bar{n}+2^{\jmath+2}-1}\right) \\
& \equiv \lambda_{2^{\jmath-1}-1} \lambda_{2^{\jmath+3} \bar{n}-2^{\jmath-1}-1} \lambda_{2^{\jmath}-1} \lambda_{2^{\jmath+2}-1} \\
& \equiv \lambda_{2^{\jmath+3} \bar{n}-2^{\jmath}-1} \lambda_{2^{j+1}-1}^{3} \\
& +\lambda_{2^{\jmath+3} \bar{n}-2^{\jmath}-2^{\jmath-1}-1} \lambda_{2^{\jmath}+2^{\jmath-1}-1} \lambda_{2^{\jmath+1}+2^{\jmath-1}} \lambda_{2^{\jmath+1}-1} \\
& +\lambda_{2^{\jmath+3} \bar{n}-2^{\jmath+1}-1} \lambda_{2^{\jmath+1}-1} \lambda_{2^{\jmath}-1} \lambda_{2^{\jmath+2}-1} \\
& +\lambda_{2^{\jmath+3} \bar{n}-2^{\jmath+1}-2^{\jmath}-2^{\jmath-1}-1} \lambda_{2^{\jmath+1}+2^{\jmath}+2^{\jmath-1}-1} \lambda_{2^{\jmath+1}+2^{\jmath}-1} \lambda_{2^{\jmath+1}-1} \\
& \bmod \Lambda\left(2^{j+3} \bar{n}-2^{j+1}-2^{j}-2^{j-1}-2\right) \text {. }
\end{aligned}
$$

We have the identities

$$
\lambda_{2^{\jmath}+2^{\jmath-1}-1} \lambda_{2^{\jmath+1}+2^{\jmath-1}}=0
$$

and

$$
\lambda_{2^{\jmath+1}+2^{\jmath}+2^{\jmath-1}-1} \lambda_{2^{\jmath+1}+2^{\jmath-1}} \lambda_{2^{\jmath+1}-1}=\lambda_{2^{\jmath-1}-1} \lambda_{2^{\jmath+2}-1}^{2}
$$

in $\Lambda$. As suggested by (2.5.5) we find

$$
\begin{aligned}
& \delta\left(\lambda_{2^{\jmath+3} \bar{n}+2^{j}-1} \lambda_{2^{j+1}-1}^{2}\right)+\delta\left(\lambda_{2^{\jmath+3} \bar{n}-2^{j+2}+2^{\jmath}-1} \lambda_{2^{\jmath+2}+2^{\jmath+1}-1^{\jmath+1}-1}\right) \\
& \quad \equiv \lambda_{2^{\jmath+3} \bar{n}-2^{\jmath}-1} \lambda_{2^{j+1}-1}^{3} \quad \bmod \Lambda\left(2^{j+3} \bar{n}-2^{j+1}-2^{j}-2^{j-1}-2\right) .
\end{aligned}
$$


It is easy to see that

$$
\begin{aligned}
& \delta\left(\lambda_{2^{\jmath+3} \bar{n}-2^{\jmath+1}-1} \lambda_{2^{\jmath+1}+2^{\jmath}-1} \lambda_{2^{\jmath+2}-1}\right) \equiv \lambda_{2^{j+3} \bar{n}-2^{\jmath+1}-1} \lambda_{2^{\jmath+1}-1} \lambda_{2^{\jmath}-1} \lambda_{2^{\jmath+2}-1} \\
& \bmod \Lambda\left(2^{j+3} \bar{n}-2^{j+1}-2^{j}-2^{j-1}-2\right) .
\end{aligned}
$$

From these equations and identities we get

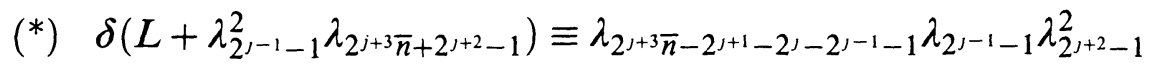

$$
\bmod \Lambda\left(2^{j+3} \bar{n}-2^{j+1}-2^{j}-2^{j-1}-2\right)
$$

where

$$
\begin{aligned}
L= & \lambda_{2^{j+3} \bar{n}+2^{\jmath-1}} \lambda_{2^{\jmath+1}-1}^{2} \\
& +\lambda_{2^{\jmath+3} \bar{n}-2^{\jmath+2}+2^{j}-1} \lambda_{2^{\jmath+2}+2^{\jmath+1}-1} \lambda_{2^{\jmath+1}-1} \\
& +\lambda_{2^{\jmath+3} \bar{n}-2^{\jmath+1}-1} \lambda_{2^{\jmath+1}+2^{\jmath}-1} \lambda_{2^{\jmath+2}-1} .
\end{aligned}
$$

Since $L \in \Lambda(i-1)\left(\right.$ recall $\left.i=2^{j+3} \bar{n}+2^{j+2}-2^{j}-1\right), L+\lambda_{2^{j-1}-1}^{2} \lambda_{2^{j+3} \bar{n}+2^{j+2}-1}$ is also a representation in $\Lambda(i)$ of $\lambda_{i} h_{j}^{2}$. From $\left(^{*}\right)$ we thus see

$$
\lambda_{i} h_{j}^{2} \rightarrow \lambda_{2^{j+3} \bar{n}-2^{\jmath+1}-2^{\jmath}-2^{\prime-1}-1} h_{j-1} h_{j+2}^{2} \text {. }
$$

So

$$
e_{i} h_{j}^{2} \rightarrow e_{2^{\jmath+3} \bar{n}-2^{\jmath+1}-2^{j}-2^{j-1}-1} h_{j-1} h_{j+2}^{2} .
$$

This proves (2.5.3).

We proceed to the proof of Theorem 1.1(4).

Proposition 2.6. The following elements in the first column are infinite cycles and are linearly independent in $E_{\infty}^{*, 3, *}$; they represent the elements of $\operatorname{Ext}_{A}^{3, *}(P)$ listed in the second column.
(1) $e_{2^{t}-1} c_{j}$
$\hat{h}_{i} c_{j}$,
$0 \leq i \leq j+1$.
(2) $e_{2^{i}-2^{\jmath+3}-2^{\jmath+1}-2 \jmath-1} c_{j+1} \longleftrightarrow \hat{h}_{i} c_{j}$,
$0 \leq j \leq i-4$.
(3) $e_{2^{i+1}+2^{i}-1} h_{i+2}^{2} h_{j} \longleftrightarrow \hat{c}_{i} h_{j}$,
$0 \leq i \leq j-4$.
(4) $e_{2^{i+1}+2^{\imath}-2^{\jmath}-1} h_{j+1} h_{i+2}^{2} \longleftrightarrow \hat{c}_{i} h_{j}$,
$0 \leq j \leq i-2$.

Proposition 2.7. The following elements in the first column are infinite cycles and are linearly independent in $E_{\infty}^{*, 3, *}$; they represent the 
elements of $\operatorname{Ext}_{A}^{3, *}(P)$ listed in the second column.
(1) $e_{2^{i}-1} h_{j}^{3}$
$\hat{h}_{i} h_{j}^{3}$,
$1 \leq i \leq j-1$.
(2) $e_{2^{i}-1} h_{j}^{2} h_{l} \longleftrightarrow \hat{h}_{i} h_{j}^{2} h_{l}$,
$1 \leq i \leq j<l-2$.
(3) $e_{2^{i}-1} h_{j} h_{k}^{2}$ $\hat{h}_{i} h_{j} h_{k}^{2}$
$1 \leq i \leq j<k-2$.
(4) $e_{2^{i}-1} h_{j} h_{k} h_{l}$
$\rightarrow \hat{h}_{i} h_{j} h_{k} h_{l}$
$1 \leq i \leq j<k-1$
$<l-2$.
(5) $e_{2^{i}-2^{\jmath+1}-2^{j}-1} h_{j+1}^{3}$
$\hat{h}_{i} h_{j}^{3}$,
$0 \leq j \leq i-3$.
(6) $e_{2^{j+3}-2^{j+1}-1} h_{j+1}^{2} h_{l}$
$\longrightarrow \hat{h}_{j+3} h_{j}^{2} h_{l}$,
$0 \leq j \leq l-4$.
(7) $e_{2^{i}-2^{j+1}-1} h_{j+1}^{2} h_{l}$ $\rightarrow \hat{h}_{i} h_{j}^{2} h_{l}$,
$0 \leq j \leq i-4 \leq l-4$.
(8) $e_{2^{i}-2^{l}-2^{j+1}-1} h_{j+1}^{2} h_{l+1}$ $\hat{h}_{i} h_{j}^{2} h_{l}$
$0 \leq j \leq l-3 \leq i-5$.
(9) $e_{2^{i}-2^{j-1}} h_{j+1} h_{k}^{2}$ $\rightarrow \hat{h}_{i} h_{j} h_{k}^{2}$
$0 \leq j \leq i-2 \leq k-2$, $j \leq k-4$.
(10) $e_{2^{i}-2^{k+1}-2^{j-1}} h_{j+1} h_{k+1}^{2} \longrightarrow \hat{h}_{i} h_{j} h_{k}^{2}$,
(11) $e_{2^{i}-2^{j}-1} h_{j+1} h_{k} h_{l} \stackrel{h_{i}}{\longrightarrow} h_{j} h_{k} h_{l}$,
$0 \leq j \leq k-3 \leq i-5$.
$0 \leq j \leq i-2 \leq k-2$,
$j \leq k-3 \leq l-5$.
(12) $e_{2^{\imath}-2^{k}-2^{\jmath}-1} h_{j+1} h_{k+1} h_{l}$ $\hat{h}_{i} h_{j} h_{k} h_{l}$,
$0 \leq j \leq k-2 \leq i-4$ $\leq l-4, k<l-2$.
(13)
$e_{2^{\imath}-2^{\prime}-2^{k}-2^{\jmath}-1} h_{j+1} h_{k+1} h_{l+1} \longleftrightarrow \hat{h}_{i} h_{j} h_{k} h_{l}$
$0 \leq j \leq k-2$
$\leq l-4 \leq i-6$.

Proposition 2.8. In $\operatorname{Ext}_{A}^{3, *}(P)$ there are the following relations.

(1) $\hat{h}_{i+3} h_{i}^{2} h_{i+3}=0, i \geq 0$.

(2) $\hat{h}_{i+2} h_{i} h_{i+3}^{2}=0, i \geq 0$.

(3) $\hat{h}_{i} c_{j}=0,0 \leq i=j+2, j+3$.

(4) $\hat{c}_{i} h_{j}=0,0 \leq i=j-3, j-2, j$ or $j+1$.

(5) $\hat{c}_{i} h_{i+1}=\hat{h}_{i+1} c_{i} \neq 0, i \geq 0$.

It is not difficult to check the following. (1) The elements listed in the first columns of (2.6) and (2.7) are not boundaries in the spectral sequence and are linearly independent. (2) Theorem 1.1(4) follows from Propositions 2.6, 2.7 and 2.8. The conclusion (1) is of course based on the above work on (2.5.1) through (2.5.16).

For the proofs of (2.6) and (2.7) we first note that 2.6(1) and 2.7(1) through $2.7(4)$ are obvious. Some of the rest follow directly from 
Proposition 2.4 by the linearity of the spectral sequence over $\mathrm{Ext}_{A}^{*, *}$ : 2.6(3) follows from 2.4(2), 2.7(6) and 2.7(7) follow from 2.4(3), 2.7(9) and 2.7(11) follow from 2.4(4), and 2.7(12) follows from 2.4(5).

What remain are 2.6(2), 2.6(4), 2.7(5), 2.7(8), 2.7(10) and 2.7(13). Except $2.6(4)$, these have a pattern in common, namely the subscripts of the factors $h_{j} h_{k} h_{l}$ or $c_{j}$ in the first columns of these rows each decreases by one when we go to the corresponding elements in the second columns. The proof for these cases is exactly the same as that for 2.4(3); one just twists one more factor in the lambda algebra representations. Note that the filtration subscripts in these cases are large enough compared to those of the attached factors $h_{j} h_{k} h_{l}$ or $c_{j}$ so that we are in the stable range and so that we can use the "trick". We leave the proof to the reader.

There is only one case left-2.6(4). The calculation for this case is also straightforward. We have the equation

$$
\begin{aligned}
& \delta\left(e_{2^{i+1}+2^{i}+2^{\jmath}-1} \lambda_{2^{i+2}-1}^{2}+e_{2^{i+1}+2^{i}-1} K\right) \\
& \equiv e_{2^{i+1}+2^{i}-1} \lambda_{2^{i+2}-1}^{2} \lambda_{2^{\jmath}-1}+e_{2^{i+1}+2^{i}-2^{\jmath}-1} \lambda_{2^{\jmath+1}-1} \lambda_{2^{i+2}-1}^{2} \\
& \bmod F\left(2^{i+1}+2^{i}-2^{j}-2\right)
\end{aligned}
$$

where $K \in \Lambda$ is an element such that

$$
\delta(K)=\lambda_{2^{j}-1} \lambda_{2^{i+2}-1}^{2}+\lambda_{2^{i+2}-1}^{2} \lambda_{2^{j-1}} .
$$

This proves 2.6(4) since $e_{2^{i+1}+2^{i}-1} \lambda_{2^{i+2}-1}^{2}$ represents $\hat{c}_{i}$.

Finally we prove Proposition 2.8. We have to show that appropriate cycle representations of the classes in (2.8) are boundaries in the differential module $\tilde{H}_{*}(P) \otimes \Lambda$. Most of the cases in (2.8) are not difficult to work out. Only two cases are rather tedious. Anyway, we will write down all the boundary homomorphism formulas, the details of which we leave to the reader to check. These formulas are derived from the differentials (2.5.1) through (2.5.16).

The case (2.8)(4) for $i=j-3$ is the simplest one since

$$
e_{2^{i+1}+2^{i}-1} \lambda_{2^{i+2}-1}^{2} \lambda_{2^{i+3}-1}=0 .
$$

Next we have the following formulas according to the case.

$$
\delta\left(e_{2^{j+2}+2^{j}-1} \lambda_{2^{j+3}-1}^{2}\right)=e_{2^{j+2}-1} \lambda_{2^{\jmath}-1} \lambda_{2^{j+3}-1}^{2} .
$$

$(2.8 .3)_{j+2}^{*} \delta\left(e_{2^{\jmath+2}+2^{\jmath+1}+2^{\jmath-1}} \lambda_{2^{j+2}-1}^{2}\right)=e_{2^{\jmath+2}-1} \lambda_{2^{\jmath+1}+2^{\jmath-1}} \lambda_{2^{\jmath+2}-1}^{2}$.

$$
\delta\left(e_{2^{j}+2^{\jmath-2}-1} \lambda_{2^{j-1}-1} \lambda_{2^{\jmath+1}-1}\right)=e_{2^{\jmath-1}+2^{\jmath-2}-1} \lambda_{2^{j}-1}^{2} \lambda_{2^{\jmath-1}} .
$$


$(2.8 .4)_{j}^{*}$

$$
\delta\left(e_{2^{j+1}+2 \jmath-1} K_{0}\right)=e_{2^{j+1}+2^{j-1}} \lambda_{2^{j+2}-1}^{2} \lambda_{2^{j-1}}
$$

where $K_{0}=\lambda_{2^{j+2}-1} \lambda_{2^{j+2}+2^{j-1}}+\lambda_{2^{j+2}+2^{j-1}} \lambda_{2^{j+2}-1}$.

$(2.8 .5)^{*}$

$$
\begin{gathered}
\delta\left(e_{2^{i+1}+2^{i}-1} \lambda_{2^{i+2}-1} \lambda_{2^{i+2}+2^{i+1}-1}\right) \\
=e_{2^{i+1}+2^{i}-1} \lambda_{2^{i+2}-1}^{2} \lambda_{2^{i+1}-1}+e_{2^{i+1}-1} \lambda_{2^{i+1}+2^{i}-1} \lambda_{2^{i+2}-1}^{2} . \\
\delta\left(\sum_{k=1}^{5} A_{k}\right)=e_{2^{i+3}-1} \lambda_{2^{i}-1}^{2} \lambda_{2^{i+3}-1}
\end{gathered}
$$

$(2.8 .1)^{*}$

where

$$
\begin{aligned}
& A_{1}=e_{2^{i+3}+2^{i}-1} \lambda_{2^{i}-1} \lambda_{2^{i+3}-1}, \\
& A_{2}=e_{2^{i+3}-2^{i}-1} \lambda_{2^{i+1}+2^{i}-1} \lambda_{2^{i+3}-1}, \\
& A_{3}=e_{2^{i+3}+2^{i+1}-1} \lambda_{2^{i+2}-1}^{2}, \\
& A_{4}=e_{2^{i+2}+2^{i}-1} \lambda_{2^{i+2}+2^{i}-1^{i}} \lambda_{2^{i+3}-1}, \\
& A_{5}=e_{2^{i+2}-1} \lambda_{2^{i+2}+2^{i+1}-1} \lambda_{2^{i+3}-1} .
\end{aligned}
$$

For 2.8(4), $i=j+1$ and $2.8(3), i=j+3$ we have only calculated the following.

$(2.8 .4)_{j+1}^{*}$

$$
\begin{array}{r}
\delta\left(e_{2^{j+1}+2^{j+1}-1} K_{1}+e_{2^{j+2}+2^{j+1}+2^{j}-1} \lambda_{2^{i+3}-1}\right) \\
\quad=e_{2^{j+2}+2^{j+1}-1} \lambda_{2^{j+3}-1}^{2} \lambda_{2^{j-1}}+e_{2^{j+2}-1} L_{1}
\end{array}
$$

where

$$
K_{1}=\lambda_{2^{\jmath+3}+2^{\jmath}-1} \lambda_{2^{j+3}-1}+\lambda_{2^{j+3}-1} \lambda_{2^{\jmath+3}+2^{j-1}}
$$

and

$$
\begin{gathered}
L_{1}=\lambda_{2^{\jmath+1}+2^{j}-1} \lambda_{2^{j+3}-1}^{2}+\lambda_{2^{\jmath+1}-1} K_{1} . \\
(2.8 .3)_{j+3}^{*} \quad \delta\left(\sum_{k=1}^{6} B_{k}\right)=e_{2^{j+3}-1} \lambda_{2^{\jmath+1}+2^{\jmath}-1} \quad \lambda_{2^{j+2}-1}^{2}+e_{2^{j+2}-1} L_{2}
\end{gathered}
$$

where $L_{2} \in \Lambda^{3,2^{j+4}-2^{j}-3}$ and

$$
\begin{aligned}
& B_{1}=e_{2^{j+3}+2^{j+1}+2^{\jmath-1}} \lambda_{2^{\jmath+2}-1}^{2}, \\
& B_{2}=e_{2^{j+3}+2^{\jmath-1}} \lambda_{2^{j+1}-1} \lambda_{2^{\jmath+3}-1}, \\
& B_{3}=e_{2^{j+2}+2^{\jmath}-1} \lambda_{2^{j+2}+2^{\jmath+1}-1} \lambda_{2^{\jmath+3}-1}, \\
& B_{4}=e_{2^{j+2}+2^{\jmath+1}-1} \lambda_{2^{j+3}+2^{\jmath}-1} \lambda_{2^{j+2}-1}, \\
& B_{5}=e_{2^{j+2}+2^{\jmath+1}-1} \lambda_{2^{j+3}-1} \lambda_{2^{\jmath+2}+2^{\jmath}-1}, \\
& B_{6}=e_{2^{j+2}+2^{j+1}-1} \lambda_{2^{j+3}+2^{j+2}-1} \lambda_{2^{j}-1} .
\end{aligned}
$$


It is clear from these two equations that $\delta\left(L_{i}\right)=0$ in $\Lambda, i=1,2$. Since $\operatorname{Ext}_{A}^{3,2^{j+4}+2^{j+1}+2^{j}}=0$ and $\operatorname{Ext}_{A}^{3,2^{j+4}-2^{\jmath}}=0$ it follows that $L_{i}$, and therefore $e_{2^{j+2}-1} L_{i}$, are boundaries and we are done.

This completes the proof of Proposition 2.8 and the whole of Theorem 1.1 .

The last piece of work in this section is to prove Theorem 2.3. The chain map

$$
\phi: \Lambda \rightarrow \tilde{H}_{*}(P) \otimes \Lambda
$$

is constructed as follows.

$\phi(1)=0$. For an admissible monomial $\lambda_{I}=\lambda_{i_{1}} \lambda_{i_{2}} \cdots \lambda_{i_{s}}$ define $\phi\left(\lambda_{0}\right)=0$ and $\phi\left(\lambda_{i_{1}}\right)=e_{i_{1}}$ for $i_{1} \geq 1$ if $s=1$. If $s \geq 2$ then $\phi\left(\lambda_{I}\right)=0$ for $i_{1}=0$ (which implies $i_{\mu}=0$ for $\mu \geq 2$ ) and $\phi\left(\lambda_{I}\right)$ for $i_{1} \geq 1$ is of the form

$$
e_{i_{1}} \lambda_{I^{\prime}}+\sum_{\nu} e_{j_{1}(\nu)} \lambda_{J^{\prime}(\nu)}
$$

where $I^{\prime}=\left(i_{2}, \ldots, i_{s}\right)$ and the second sum is described as follows. First we require each $J^{\prime}(\nu)=\left(j_{2}(\nu), \ldots, j_{s}(\nu)\right)$ be admissible and $J(\nu)=$ $\left(j_{1}(\nu), j_{2}(\nu), \ldots, j_{s}(\nu)\right)$ be inadmissible. Secondly, choose any large integer $m$ (compared to $i_{j}$ and $s$ ) and let

$$
J(\nu, m)=\left(2^{m}+j_{1}(\nu), j_{2}(\nu), \ldots, j_{s}(\nu)\right)
$$

which is admissible. Then $e_{j_{1}(\nu)} \lambda_{J^{\prime}(\nu)}$ appears in the second sum of $\left(^{*}\right)$ if and only if, for some $q \geq 2, \lambda_{J(\nu, m)}$ appears in the admissible expansion of

$$
\lambda_{i_{1}} \cdots \lambda_{i_{q-1}} \lambda_{i_{q}+2^{m}} \lambda_{i_{q+1}} \cdots \lambda_{i_{s}}
$$

It is proved in [17] that the second sum of $\left(^{*}\right)$ is independent of the large integer $m$ chosen and that the map $\phi$ defined above is a chain map. To complete the proof of Theorem 2.3 it suffices to show $j_{1}(\nu)<i_{1}$ for each $\nu$. In the proof for Lemma 3.2 in [17] the following identity is obtained:

$$
j_{1}(\nu)=i_{q}-i_{q-1}-\cdots-i_{1}-\mu_{q-1}-\mu_{q-2}-\cdots-\mu_{1}-q+1
$$

for some non-negative integers $\mu_{t}$. Thus

$$
j_{1}(\nu) \leq i_{q}-i_{q-1}-\cdots-i_{1}-q+1
$$


Since $\left(i_{1}, i_{2}, \ldots, i_{q}\right)$ is admissible, $i_{1} \geq 2 i_{2}, i_{2} \geq 2 i_{3}, \ldots, i_{q-1} \geq 2 i_{q}$ which imply $i_{1} \geq i_{q} / 2^{q-1}, i_{2} \geq i_{q} / 2^{q-2}, \ldots, i_{q-1} \geq i_{q} / 2$. Then

$$
\begin{aligned}
i_{1}- & j_{1}(\nu) \geq i_{1}-\left(i_{q}-i_{q-1}-\cdots-i_{2}-i_{1}-q+1\right) \\
& =\left(2 i_{1}+i_{2}+\cdots+i_{q-1}\right)-i_{q}+q-1 \\
& \geq i_{q}\left(\frac{1}{2^{q-2}}+\frac{1}{2^{q-2}}+\frac{1}{2^{q-3}}+\cdots+\frac{1}{2}\right)-i_{q}+q-1 \\
& =i_{q}-i_{q}+q-1=q-1 \geq 1>0
\end{aligned}
$$

since $q \geq 2$. This proves Theorem 2.3.

3. Proof of Theorem 1.3. We begin by recalling the following result proved in [12].

(3.1) For each $i \geq 0$ there is a stable map $f_{i}: S^{2^{i+1}} \rightarrow P$ such that in the mapping cone $X=P \cup_{f_{i}} e^{2^{i+1}+1}$ the Steenrod operation

$$
\mathrm{Sq}^{2^{i+1}}: H^{1}(X)=\mathrm{Z} / 2 \rightarrow H^{2^{i+1}+1}(X)=\mathrm{Z} / 2
$$

is non-zero.

This is Theorem 1.3(1). We will infer from this Theorem 1.3(3).

Let $\Phi_{i, i}$ be the secondary cohomology operation associated with the Adem relation

$$
\mathrm{Sq}^{2 i} \mathrm{Sq}^{2^{i}}+\sum_{k=2}^{i-1} \mathrm{Sq}^{2^{i+1}-2^{k}} \mathrm{Sq}^{2^{k}}+\mathrm{Sq}^{4} \mathrm{Sq}^{2^{i+1}-4}=0, \quad i \geq 2 .
$$

$\Phi_{i, i}$ is defined on any cohomology class of a space on which the Steenrod operations $\mathrm{Sq}^{l}$ are zero for $l \geq 4$. From (3.1) and Adams's Hopf invariant Theorem ([2]) we have the following.

(3.2) $\Phi_{i, i}: H^{2}(X)=\mathbf{Z} / 2 \rightarrow H^{2^{1+1}+1}(X)=\mathbf{Z} / 2$ is non-zero for $i \geq 3$.

Proposition 3.3. There is a stable map $f: \Sigma P \rightarrow P$ such that $f^{*}: H^{2 k+1}(P)=\mathbf{Z} / 2 \rightarrow H^{2 k+1}(\Sigma P)=H^{2 k}(P)=\mathbf{Z} / 2$ is an isomorphism, $k \geq 1$.

There are several ways to see this. Perhaps, the simplest way now is the following. Let $\mathbf{C P}=\mathbf{C} P^{\infty}$ be the infinite complex projective space. It is proved in [23] that there is a stable map $g: \Sigma C P \rightarrow P$ such that $g^{*}: H^{*}(P) \rightarrow H^{*}(\Sigma \mathrm{C} P)$ is onto. Let $\pi: P \rightarrow \mathbf{C P}$ be the standard map. Then $f=g \circ(\Sigma \pi): \Sigma P \rightarrow P$ has the property in (3.3). 
Taking the composite $\varphi_{i}=f \circ \Sigma f_{i}: S^{2^{i+1}} \rightarrow P$ of the maps in (3.1) and (3.3) we see from (3.2) the following.

In the mapping cone $Y=P \cup_{\varphi_{l}} e^{2^{i+1}+2}$ the secondary cohomology operation

$$
\Phi_{i, i}: H^{3}(Y)=\mathrm{Z} / 2 \rightarrow H^{2^{i+1}+2}(Y)=\mathrm{Z} / 2
$$

is non-zero for $i \geq 3$.

In the Adams spectral sequence setting this means that $\varphi_{i}$ is detected by $\hat{h}_{2} h_{i}^{2} \in \operatorname{Ext}_{A}^{2, *}(P)$. This proves Theorem 1.3(3) for $i \geq 3$. The case $i=2$ is trivial.

The following theorem is the main topological result of the paper from which the remaining cases of Theorem 1.3 are proved. To state it, let $B(k)$ be the $k$ th 2-primary Brown-Gitler spectrum ([7]). Recall $H^{*}(B(k)) \cong A / A\left\{\chi\left(\mathrm{Sq}^{i}\right) \mid i>k\right\}$ which has $\left\{\chi\left(\mathrm{Sq}^{I}\right) \mid I=\left(i_{1}, \ldots, i_{s}\right)\right.$ admissible, $\left.i_{1} \leq k\right\}$ a $\mathrm{Z} / 2$-base where $\chi: A \rightarrow A$ is the canonical antiautomorphism of the Steenrod algebra $A$. In particular $H^{l}\left(B\left(2^{i}\right)\right)=0$ for $l>2^{i+1}-1$ and

$$
\text { (a) } \mathrm{Sq}^{1}: H^{2^{i+1}-2}\left(B\left(2^{i}\right)\right)=\mathrm{Z} / 2 \rightarrow H^{2^{i+1}-1}\left(B\left(2^{i}\right)\right)=\mathrm{Z} / 2
$$

is non-zero.

THEOREM 3.5. For each $i \geq 1$ there is a stable map $f_{i}: \Sigma^{2^{i}} B\left(2^{i-1}\right)$ $\rightarrow P$ such that $f_{i}^{*}: H^{2^{i+1}-1}(P)=\mathrm{Z} / 2 \rightarrow H^{2^{i+1}-1}\left(\Sigma^{2^{i}} B\left(2^{i-1}\right)\right)=\mathrm{Z} / 2$ is non-zero and is zero in other dimensions.

We prove this later. To prove $(1.3)(2),(4)$ and (5) we derive from (3.5) a corollary and recall some results from [12], [18] and [22].

Let $X_{i}=\left[\Sigma^{2^{i}} B\left(2^{i-1}\right)\right]^{2^{+1}-2}, \bar{f}_{i}=f_{i} \mid X_{i}$ and $Y_{i}=P \cup_{\bar{f}_{i}} C X_{i}$. Then there is a short exact sequence

$$
0 \leftarrow H^{*}(P) \stackrel{j^{*}}{\leftarrow} H^{*}\left(Y_{i}\right) \stackrel{q^{*}}{\leftarrow} H^{*}\left(\Sigma X_{i}\right) \leftarrow 0
$$

where $j: P \rightarrow Y_{i}$ is the inclusion map and $q: Y_{i} \rightarrow \Sigma X_{i}$ is the pinching map. Recall $H^{*}(P)=\mathbf{Z} / 2[x]$. Since $j^{*}$ is isomorphism in dimensions $\leq 2^{i}, H^{2^{i}-1}\left(Y_{i}\right) \cong \mathrm{Z} / 2$ is generated by $x^{2^{i}-1}$. Let $y_{i} \in H^{2^{i+1}-1}\left(Y_{i}\right)$ be the image of the non-zero class in $H^{2^{i+1}-1}\left(\Sigma X_{i}\right)=\mathrm{Z} / 2$ under $q^{*}$.

Corollary 3.6. In $H^{*}\left(Y_{i}\right), \operatorname{Sq}^{2^{i}}\left(x^{2^{i}-1}\right)=y_{i}$ for $i \geq 3$.

This follows from (a), Theorem 3.5 and Adams's differentials $d_{2}\left(\hat{h}_{i+1}\right)=\hat{h}_{i} h_{i} h_{0}, i \geq 3$ in the ASS for $P$. 
We recall the following:

(3.7) ([22]) There is a map $g_{1}: S^{2^{i+1}} \rightarrow \Sigma^{2^{i}} B\left(2^{i-1}\right)$ for each $i \geq 1$ such that

$$
\begin{aligned}
\mathrm{Sq}^{2}: & H^{2^{i+1}-1}\left(\Sigma^{2^{i}} B\left(2^{i-1}\right) \cup_{g_{1}} e^{2^{i+1}+1}\right) \\
& =\mathrm{Z} / 2 \rightarrow H^{2^{i+1}+1}\left(\Sigma^{2^{i}} B\left(2^{i-1}\right) \cup_{g_{1}} e^{2^{i+1}+1}\right)=\mathrm{Z} / 2
\end{aligned}
$$

is non-zero.

(3.8) ([12]) There is a map $g_{2}: S^{2^{i+1}+1} \rightarrow X_{i}$ for each $i \geq 1$ such that

$$
\begin{aligned}
\mathrm{Sq}^{4}: & H^{2^{i+1}-2}\left(X_{i} \cup_{g_{2}} e^{2^{i+1}+2}\right) \\
& =\mathrm{Z} / 2 \rightarrow H^{2^{i+1}+2}\left(X_{i} \cup_{g_{2}} e^{2^{i+1}+2}\right)=\mathbf{Z} / 2
\end{aligned}
$$

is non-zero.

(3.9) ([18]) There is a map $g_{3}: S^{2^{i+1}+18} \rightarrow \Sigma^{2^{i}} B\left(2^{i-1}\right)$ for each $i \geq 1$ such that the composite

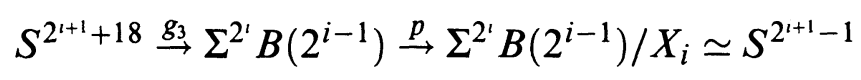

is detected by $c_{1} \in \mathrm{Ext}_{A}^{3, *}$ in the Adams spectral sequence for ${ }_{2} \pi_{*}^{s}$.

It follows from (3.5), (3.6), (3.7), (3.8) and (3.9) that the maps

$$
\begin{gathered}
f_{i} \circ g_{1}: S^{2^{i+1}} \rightarrow P, \\
\bar{f}_{i} \circ g_{2}: S^{2^{i+1}+1} \rightarrow P,
\end{gathered}
$$

and

$$
f_{i} \circ g_{3}: S^{2^{i+18}} \rightarrow P
$$

are detected respectively by $\hat{h}_{i} h_{1}(i \geq 3), \hat{h}_{i} h_{i} h_{2}(i \geq 5)$ and $\hat{h}_{i} c_{1}$ $(i \neq 3,4)$. This proves Theorem 1.3.

To prove Theorem 3.5, consider the double loop space $\Omega^{2} S^{3}$. There is a 2-primary stable decomposition

$$
\Omega^{2} S^{3} \simeq \bigvee_{k=1}^{\infty} \Sigma^{k} B\left(\left[\frac{k}{2}\right]\right)
$$

This is due to Brown-Peterson, Mahowald and Snaith ([8], [22], [26]) (odd primary analogues are proved in [11]). We will consider a composite

$$
\text { (b) } f: \Omega^{2} S^{3} \stackrel{\gamma}{\rightarrow} B O \stackrel{\sigma}{\rightarrow} B O(2) \stackrel{t}{\rightarrow} P \times P \stackrel{\pi}{\rightarrow} P
$$

of stable maps and show that for each $i \geq 1, f_{i}=f \mid \Sigma^{2^{t}} B\left(2^{i-1}\right)$ is the map we want. Here $B O(n)$ is the classifying space for the orthogonal 
group $O(n), O=O(\infty)$. Note that $P=B O(1)$. The four maps $\gamma, \sigma, t$ and $\pi$ are described as follows.

$\gamma$ and $\pi$ are maps of spaces. $\pi$ is the first factor projection map (any factor will do). To describe $\gamma$, let $\eta: S^{1} \rightarrow B O$ be a map representing the generator of $\pi_{1}(B O)=Z / 2$. $B O$ is an infinite loop space. In particular, $B O=\Omega^{2} X$ for some space $X$. Let $\eta^{*}: S^{3} \rightarrow X$ be the adjoint of $\eta$. Then $\gamma=\Omega^{2} \eta^{*}: \Omega^{2} S^{3} \rightarrow \Omega^{2} X=B O$.

V. P. Snaith shows in [27] that there is a stable decomposition

$$
B O \simeq \bigvee_{n=1}^{\infty} \frac{B O(2 n)}{B O(2 n-2)}
$$

Then $\sigma: B O \rightarrow B O(2)$ is the projection to the first wedge summand $B O(2)$. $t: B O(2) \rightarrow B O(1) \times B O(1)=P \times P$ is the Becker-Gottlieb transfer map to the fibration $O(2) / O(1) \times O(1) \rightarrow B O(1) \times B O(1) \stackrel{p}{\rightarrow}$ $B O(2)([5])$. Note that $\sigma$ and $t$ are defined only as stable maps.

To take the composite $f$ in (b) we stabilize $\gamma$ and $\pi$ also.

Recall that $H_{*}\left(\Omega^{2} S^{3}\right) \cong \mathrm{Z} / 2\left[\xi_{1}, \xi_{2}, \ldots\right],\left|\xi_{k}\right|=2^{k}-1$ ([10]). Assign to each monomial $\xi_{1}^{i_{1}} \cdots \xi_{n}^{i_{n}}$ the filtration $\Sigma_{k=1}^{n} i_{k} 2^{k-1}$. Then the wedge summand $\Sigma^{2^{i}} B\left(2^{i-1}\right)$ of $\Omega^{2} S^{3}$ has the set of all monomials of filtration $2^{i}$ as a $\mathbf{Z} / 2$-base for its homology $H_{*}\left(\Sigma^{2^{i}} B\left(2^{i-1}\right)\right)$ ([24]). In particular, $H_{2^{i+1}-2}\left(\Sigma^{2^{i}} B\left(2^{i-1}\right)\right)=\mathrm{Z} / 2$ is generated by $\xi_{i}^{2}$ and $H_{2^{i+1}-1}\left(\Sigma^{2^{i}} B\left(2^{i-1}\right)\right)$ $=\mathrm{Z} / 2$ is generated by $\xi_{i+1}$. It suffices to show the following.

PROPOSITION 3.10. (1) $f_{*}=\pi_{*} t_{*} \sigma_{*} \gamma_{*}: H_{*}\left(\Omega^{2} S^{3}\right)=\mathbf{Z} / 2\left[\xi_{1}, \xi_{2}, \ldots\right]$ $\rightarrow H_{*}(P)$ is zero on monomials of length $\geq 3$.

(2) $f_{*}\left(\xi_{i}^{2}\right)=0$.

(3) $f_{*}\left(\xi_{i+1}\right)=e_{2^{i+1}-1}$.

To prove this we recall some facts about the mod 2 cohomology and homology of $B O$ and $B O(2)$.

First, $H^{*}(B O)=\mathbf{Z} / 2\left[w_{1}, w_{2}, \ldots\right]$ and $H^{*}(B O(2))=\mathbf{Z} / 2\left[w_{1}, w_{2}\right]$ where $w_{i}$ are the universal Stiefel-Whitney classes. If $j: B O(2) \rightarrow B O$ is the inclusion map then $j^{*}\left(w_{i}\right)=0$ for $i \geq 3$ and $j^{*}\left(w_{i}\right)=w_{i}$ for $i=1,2$. In particular, $j^{*}$ is onto. Induced by the loop multiplication $\mu: B O \times B O \rightarrow B O$ is the diagonal $\Delta=\mu^{*}$ in the Hopf algebra $H^{*}(B O)$ 
with $\Delta\left(w_{i}\right)=\sum_{k=0}^{i} w_{k} \otimes w_{i-k}\left(w_{0}=1\right)$. Let $\bar{e}_{i} \in H_{i}(B O)$ be dual to $w_{1}^{i}$ in the monomial basis of $H^{*}(B O)$. Then $H_{*}(B O)=\mathbf{Z} / 2\left[\bar{e}_{1}, \bar{e}_{2}, \ldots\right]$. If $H_{*}(B O(2))$ is identified with $j_{*} H_{*}(B O(2)) \subset H_{*}(B O)$ then $H_{*}(B O(2))=\mathbf{Z} / 2$-submodule generated by monomials in the $\bar{e}_{j}$ 's of length $\leq 2$. The following is easy to prove; it will be needed in the proof of $3.10(2)$ and 3.10(3) later.

(3.11) $\left\langle\bar{e}_{2^{i}-1}^{2}, W\right\rangle=0$ for any monomial $W \in H_{*}(B O(2))$ except $W=w_{2}^{2^{i}-1}$, in which case $\left\langle\bar{e}_{2^{i}-1}^{2}, w_{2}^{2^{i}-1}\right\rangle=1$.

Snaith shows in [27] the following.

(3.12) The induced map $\sigma_{*}: H_{*}(B O)=\mathbf{Z} / 2\left[\bar{e}_{1}, \bar{e}_{2}, \ldots\right] \rightarrow$ $H_{*}(B O(2))$ of the stable map $\sigma: B O \rightarrow B O(2)$ is the identity map on $H_{*}(B O(2))$ and is zero on any monomial of length $\geq 3$.

This implies 3.10(1) since $\gamma_{*}: H_{*}\left(\Omega^{2} S^{3}\right) \rightarrow H_{*}(B O)$ is a ring homomorphism.

In

$$
H_{*}\left(\Omega^{2} S^{3}\right)=\mathbf{Z} / 2\left[\xi_{1}, \xi_{2}, \ldots\right], \quad \xi_{i}=Q_{1}^{i-1} \xi_{1}=\underset{i-1}{Q_{1} \ldots Q_{1}} \xi_{1}
$$

for $i \geq 1$ where $Q_{1}: H_{l}\left(\Omega^{2} S^{3}\right) \rightarrow H_{2 l+1}\left(\Omega^{2} S^{3}\right)$ is the Dyer-Lashof operation ([10]). By the construction of $\gamma, \gamma_{*}\left(\xi_{1}\right)=\bar{e}_{1}$. Since $\gamma$ is a double loop map it follows that $\gamma_{*}\left(\xi_{i}\right)=\gamma_{*}\left(Q_{1}^{i-1} \xi_{1}\right)=Q_{1}^{i-1} \gamma_{*}\left(\xi_{1}\right)=$ $Q_{1}^{i-1} \bar{e}_{1}$. Kochman proves in [16] the following.

(3.13) (1) $\gamma_{*}\left(\xi_{i}\right)=Q_{1}^{i-1} \bar{e}_{1} \in H_{2^{i}-1}(B O)$ is the element dual to $w_{2^{i}-1}$ in the monomial basis of $H^{*}(B O)=\mathbf{Z} / 2\left[w_{1}, w_{2}, \ldots\right]$ for $i \geq 1$.

(2) $\gamma_{*}\left(\xi_{i}\right)=Q_{1}^{i-1} \bar{e}_{1}=\bar{e}_{2^{i}-1}+$ decomposables, $i \geq 1$. (3.12) and (3.13)(2) imply the following.

$$
\sigma_{*} \gamma_{*}\left(\xi_{i}^{2}\right)=\bar{e}_{2^{i}-1}^{2}, \quad i \geq 1
$$

Recall that $H^{*}(P)=\mathrm{Z} / 2[x]$ and $H^{*}(P \times P)=\mathrm{Z} / 2\left[x_{1}, x_{2}\right]$. Then $\pi^{*}\left(x^{2^{i+1}-2}\right)=x_{1}^{2^{i+1}-2}$ and $\pi^{*}\left(x^{2^{i+1}-1}\right)=x_{1}^{2^{i+1}-1}$. Feshbach shows in [14] that the induced map $p^{*} t^{*}: H^{*}(P \times P) \rightarrow H^{*}(P \times P)$ of the composite

$$
P \times P=B O(1) \times B O(1) \stackrel{p}{\rightarrow} B O(2) \stackrel{t}{\rightarrow} P \times P
$$


maps $x_{1}^{2^{i+1}-2}$ to $x_{1}^{2^{i+1}-2}+x_{2}^{2^{i+1}-2}$ and $x_{1}^{2^{i+1}-1}$ to $x_{1}^{2^{i+1}-1}+x_{2}^{2^{i+1}-1}$. The ring homomorphism $p^{*}: H^{*}(B O(2))=\mathbf{Z} / 2\left[w_{1}, w_{2}\right] \rightarrow H^{*}(P \times P)=$ $\mathbf{Z} / 2\left[x_{1}, x_{2}\right]$ mapping $w_{1}$ to $x_{1}+x_{2}$ and $w_{2}$ to $x_{1} x_{2}$ is monomorphic with im $p^{*}=$ the subring consisting of all the symmetric polynomials in $x_{1}$ and $x_{2}$. One calculates then the following.

$$
\begin{aligned}
& \text { (1) } t^{*} \pi^{*}\left(x^{2^{i+1}-2}\right)=\sum_{k=0}^{i-1} w_{1}^{2^{i+1}-2^{k+2}+2} w_{2}^{2^{k+1}-2} . \\
& \text { (2) } t^{*} \pi^{*}\left(x^{2^{i+1}-1}\right)=w_{1}^{2^{1+1}-1}+w_{1} w_{2}^{2^{i}-1}+\sum_{k=0}^{i-1} w_{1}^{2^{i+1}-2^{k+1}+1} w_{2}^{2^{k}-1} .
\end{aligned}
$$

Note that the monomial $w_{2}^{2^{i}-1}$ does not appear in the expansion of $t^{*} \pi^{*}\left(x^{2^{i+1}-2}\right)$ in (3.15)(1). Thus from (3.11) and (3.14) we see $f_{*}\left(\xi_{i}^{2}\right)=$ 0 . This proves $3.10(2)$.

To prove $3.10(3)$ we note that the result is trivially true for $i=0$. So assume $i \geq 1$. We will show the following.

$$
\begin{aligned}
& \text { (1) } \gamma^{*} \sigma^{*}\left(w_{1}^{2^{i+1}-1}\right)=0 . \\
& \text { (2) } \gamma^{*} \sigma^{*}\left(w_{1} w_{2}^{2^{i}-1}\right) \neq 0 . \\
& \text { (3) } \gamma^{*} \sigma^{*}\left(w_{1}^{2^{i+1}-2^{k+1}+1} w_{2}^{2^{k}-1}\right)=0 \text { for } 1 \leq k \leq i-1 .
\end{aligned}
$$

This together with $(3.15)(2)$ implies $f^{*}\left(x^{2^{1+1}-1}\right) \neq 0$ in

$$
H^{2^{i+1}-1}\left(\Sigma^{2^{i}} B\left(2^{i-1}\right)\right)
$$

and this is $3.10(3)$.

To show (3.16)(2) and (3) we recall that in $H^{*}(B O(2)), \mathrm{Sq}^{1} w_{2}=$ $w_{1} w_{2}$. Then

$$
\mathrm{Sq}^{1}\left(w_{2}^{2^{i}-1}\right)=w_{1} w_{2}^{2^{i}-1}
$$

and

$$
\mathrm{Sq}^{1}\left(w_{1}^{2^{i+1}-2^{k+1}} w_{2}^{2^{k}-1}\right)=w_{1}^{2^{2+1}-2^{k+1}+1} w_{2}^{2^{k}-1} \text { for } 1 \leq k \leq i-1 .
$$

By (3.11) and (3.14), $\gamma^{*} \sigma^{*}\left(w_{2}^{2^{t}-1}\right) \neq 0$ and $\gamma^{*} \sigma^{*}\left(w_{1}^{2^{i+1}-2^{k+1}} w_{2}^{2^{k}-1}\right)=0$ for $1 \leq k \leq i-1$ in $H^{2^{i+1}}\left(\Sigma^{2^{i}} B\left(2^{i-1}\right)\right)=Z / 2$. Since

$$
\begin{aligned}
\mathrm{Sq}^{1}: & H^{2^{i+1}-2}\left(\Sigma^{2^{i}} B\left(2^{i-1}\right)\right) \\
& =\mathrm{Z} / 2 \rightarrow H^{2^{i+1}-1}\left(\Sigma^{2^{i}} B\left(2^{i-1}\right)\right)=\mathrm{Z} / 2
\end{aligned}
$$


is non-zero it follows that $\gamma^{*} \sigma^{*}\left(w_{1} w_{2}^{2^{i}-1}\right) \neq 0$ and

$$
\gamma^{*} \sigma^{*}\left(w_{2}^{2^{i+1}-2^{k+1}+1} w_{2}^{2^{k}-1}\right)=0 \text { for } 1 \leq k \leq i-1 .
$$

This proves $3.16(2)$ and (3).

We prove $3.16(1)$ by contradiction. Suppose $\gamma^{*} \sigma^{*}\left(w_{1}^{2^{i+1}-1}\right) \neq 0$. By (3.12) $\sigma^{*}\left(w_{1}^{2^{i+1}-1}\right)=w_{1}^{2^{i+1}-1}+\sum_{l \geq 1} W_{l}$ where each $W_{l}$ is a monomial in $\operatorname{ker} j^{*}$, the ideal of $H^{*}(B O)$ generated by $w_{3}, w_{4}, \ldots$ Since $\gamma^{*} \sigma^{*}\left(w_{1}^{2^{2+1}-1}\right) \neq 0$ in $H^{2^{i+1}-1}\left(\Sigma^{2^{i}} B\left(2^{i-1}\right)\right)=\mathrm{Z} / 2$, by $3.13(1)$ we see one of the monomials $W_{l}$ must be $w_{2^{i+1}-1}\left(\right.$ since $2^{i+1}-1 \geq 3$ ), say $W_{1}=w_{2^{i+1}-1}$. Thus $\sigma^{*}\left(w_{1}^{2^{i+1}-1}\right)=w_{1}^{2^{l+1}-1}+w_{2^{l+1}-1}+\sum_{l \geq 2} W_{l}$ where, for $l \geq 2, W_{l} \neq w_{2^{i+1}-1}$.

Recall that in $H^{*}(B O), \mathrm{Sq}^{1} w_{2 n}=w_{2 n+1}+w_{1} w_{2 n}(n \geq 1)$ and $\mathrm{Sq}^{1} w_{2 n+1}=w_{1} w_{2 n+1}(n \geq 0)$. In particular, $\mathrm{Sq}^{1} w_{1}^{2^{i+1}-1}=w_{1}^{2^{i+1}}$ and $\mathrm{Sq}^{1} w_{2^{i+1}-1}=w_{1} w_{2^{i+1}-1}$. Also, for any monomial $W \in H^{2^{i+1}-1}(B O)$ other than $w_{2^{1+1}-1}, w_{1} w_{2^{i+1}-1}$ does not appear in the expansion of $\mathrm{Sq}^{1} W$ as sum of monomials. So

$$
\mathrm{Sq}^{1} \sigma^{*}\left(w_{1}^{2^{l+1}-1}\right)=w_{1}^{2^{i+1}}+w_{1} w_{2^{i+1}-1}+\mathrm{Sq}^{1}\left(\sum_{l \geq 2} W_{l}\right) \neq w_{1}^{2^{i+1}}
$$

On the other hand, since $\sigma^{*}$ commutes with the Steenrod operations, we see

$$
\begin{aligned}
\mathrm{Sq}^{1} \sigma^{*}\left(w_{1}^{2^{i+1}-1}\right) & =\sigma^{*}\left(\mathrm{Sq}^{1} w_{1}^{2^{i+1}-1}\right)=\sigma^{*}\left(w_{1}^{2^{i+1}}\right) \\
& =\sigma^{*}\left(\mathrm{Sq}^{2^{i}} \mathrm{Sq}^{2^{i-1}} \cdots \mathrm{Sq}^{2} \mathrm{Sq}^{1} w_{1}\right) \\
& =\mathrm{Sq}^{2^{i}} \mathrm{Sq}^{2^{i-1}} \cdots \mathrm{Sq}^{2} \mathrm{Sq}^{1} \sigma^{*}\left(w_{1}\right) \\
& =\mathrm{Sq}^{2^{i}} \mathrm{Sq}^{2^{i-1}} \cdots \mathrm{Sq}^{2} \mathrm{Sq}^{1} w_{1}=w_{1}^{2^{i+1}} .
\end{aligned}
$$

This contradiction proves $3.16(1)$.

This completes the proof of Proposition 3.10 and therefore of Theorem 3.5 .

\section{REFERENCES}

[1] J. F. Adams, On the structure and applications of the Steenrod algebra, Comm. Math. Helv., 32 (1958), 180-214.

[2] On the non-existence of elements of Hopf invariant one, Ann. of Math., 72 (1960), 20-104.

[3] M. G. Barratt, J. D. S. Jones and M. E. Mahowald, The Kervaire invariant problem, Proc. Northwestern Homotopy Theory Conference, Contemporary Math. Amer. Math. Soc., 19 (1983), 9-23.

[4] Relations amongst Toda brackets and the Kervaire invariant in dimension 62, J. London Math. Soc., 30 (1984), 533-550. 
[5] J. C. Becker and D. H. Gottlieb, The transfer map and fiber bundles, Topology, 14 (1975), 1-12.

[6] A. K. Bousfield, E. B. Curtis, D. M. Kan, D. G. Quillen, D. L. Roctor and J. W. Schlesinger, The mod- $p$ lower central series and the Adams spectral sequence, Topology, 5 (1966), 331-342.

[7] E. H. Brown, Jr. and S. Gitler, A spectrum whose cohomology is certain cyclic module over the Steenrod algebra, Topology, 12 (1973), 283-296.

[8] E. H. Brown, Jr. and F. P. Peterson, On the stable decomposition of $\Omega^{2} S^{r+2}$, Trans. Amer. Math. Soc., 243 (1978), 287-298.

[9] R. Bruner, An infinite family in $\pi * S^{0}$ derived from Mahowald's $\eta_{\text {, family, Proc. }}$ Amer. Math. Soc., 82 (1981), 637-639.

[10] F. R. Cohen, T. L. Lada and J. P. May, The Homology of Iterated Loop Spaces, Lec. Notes in Math. No. 533, Springer-Verlag, New York (1976).

[11] R. L. Cohen, Odd primary infinite families in stable homotopy theory, Memo. Amer. Math. Soc., No. 242 (1981).

[12] R. L. Cohen, J. D. S. Jones and Mark E. Mahowald, The Kervaire invariant of immersions, Invent. Math., 79 (1985), 95-123.

[13] E. B. Curtis, Simplicial homotopy theory, Advances in Math., 6 (1971), 107207.

[14] M. Feshbach, The transfer and compact Lie groups, Trans. Amer. Math. Soc., 251 (1979), 139-169.

[15] D. S. Kahn and S. B. Priddy, Applications of the transfer to the stable homotopy theory, Bull. Amer. Math. Soc., 78 (1972), 981-991.

[16] S. O. Kochman, Homology of the classical groups over the Dyer-Lashof operations, Trans. Amer. Math. Soc., 185 (1973), 83-136.

[17] W. H. Lin, Algebraic Kahn-Priddy theorem, Pacific J. Math., 96 (1981), 435455.

[18] Some elements in the stable homotopy of spheres, Proc. Amer. Math. Soc., 95 (1985), 295-298.

[19] M. E. Mahowald, The metastable homotopy of $S^{n}$, Memo. Amer. Math. Soc., 72 (1967).

[20] M. E. Mahowald and M. Tangora, Some differentials in the Adams spectral sequence, Topology, 6 (1967), 349-369.

[21] - On secondary operations which detect homotopy classes, Boletin Soc. Mat. Mexi., (1967), 71-75.

[22] M. E. Mahowald, $A$ new infinite family in ${ }_{2} \pi_{*}^{s}$, Topology, 16 (1977), 249-256.

[23] B. M. Mann, E. Y. Miller and H. R. Miller, $S^{1}$-equivariant function spaces and characteristic classes, Trans. Amer. Math. Soc., 295 (1986), 233-256.

[24] R. J. Milgram, Iterated loop spaces, Ann. of Math., 84 (1966), 386-403.

[25] D. Ravenel, Complex Cobordism and Stable Homotopy of Spheres, Academic Press, 1986.

[26] V. P. Snaith, A stable decomposition for $\Omega^{n} S^{n} X$, J. London Math. Soc., 2 (1974), 577-583.

[27] _ Algebraic cobordism and K-theory, Memo. Amer. Math. Soc., No. 221 (1979).

[28] J. S. P. Wang, On the cohomology of the mod 2 Steenrod algebra and nonexistence of elements of Hopf invariant one, Illinois J. Math., 11 (1967), 480490. 
Received November 17, 1986. Cohen and Mahowald were supported by N.S.F. grants, and Cohen by an N.S.F.-P.Y.I. grant. Lin was supported by the N.S.C. of the R.O.C. and also by a China Foundation fellowship.

STANFORD UNIVERSITY

STANFORD, CA 94305

National Tsing Hua University

HSINChU, TaIwaN

AND

NORTHWESTERN UNIVERSITY

EvaNSTON, IL 60201 



\section{PACIFIC JOURNAL OF MATHEMATICS EDITORS}

V. S. VARADARAJAN (Managing Editor) University of California Los Angeles, CA 90024

HeRbert Clemens

University of Utah

Salt Lake City, UT 84112

R. FINN

Stanford University

Stanford, CA 94305
ROBION KIRBY

University of California

Berkeley, CA 94720

C. C. MOORE

University of California

Berkeley, CA 94720

HAROLD STARK

University of California, San Diego

La Jolla, CA 92093

\section{ASSOCIATE EDITORS}
R. ARENS
E. F. BECKENBACH
B. H. NEUMANN
F. Wolf
K. YOSHIDA (1906-1982)

\section{SUPPORTING INSTITUTIONS}

UNIVERSITY OF ARIZONA UNIVERSITY OF OREGON

UNIVERSITY OF BRITISH COLUMBIA UNIVERSITY OF SOUTHERN CALIFORNIA

CALIFORNIA INSTITUTE OF TECHNOLOGY UNIVERSITY OF CALIFORNIA MONTANA STATE UNIVERSITY STANFORD UNIVERSITY

UNIVERSITY OF NEVADA, RENO UNIVERSITY OF HAWAII NEW MEXICO STATE UNIVERSITY UNIVERSITY OF TOKYO UNIVERSITY OF UTAH OREGON STATE UNIVERSITY WASHINGTON STATE UNIVERSITY UNIVERSITY OF WASHINGTON 


\section{Pacific Journal of Mathematics}

Vol. 134, No. $1 \quad$ May, 1988

Marco Abate, Annular bundles . ............................

Ralph Cohen, Wen Hsiung Lin and Mark Mahowald, The Adams

spectral sequence of the real projective spaces

Harry Joseph D'Souza, Threefolds whose hyperplane sections are elliptic surfaces .........................................57

Theodore Gerard Faticoni, Localization in finite-dimensional FPF rings . . . 79

Daniel Hitt, Invariant subspaces of $\mathscr{H}^{2}$ of an annulus ................ 101

Ellen Kirkman and James J. Kuzmanovich, On the global dimension of fibre products

Angel Rafael Larotonda and Ignacio Zalduendo, Homogeneous spectral sets and local-global methods in Banach algebras

Halsey Lawrence Royden, Jr., Invariant subspaces of $\mathscr{H}^{p}$ for multiply

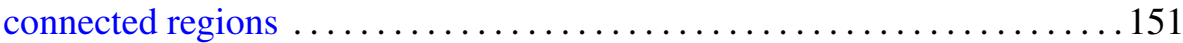

Jane Sangwine-Yager, A Bonnesen-style inradius inequality in 3-space . . . . 173

Stefano Trapani, Holomorphically convex compact sets and cohomology

Thomas Vogel, Uniqueness for certain surfaces of prescribed mean

curvature 